\title{
Nonlinear perturbations of cosmological scalar fields with non-standard kinetic terms
}

\author{
Sébastien Renaux-Petel ${ }^{a 1}$ and Gianmassimo Tasinato ${ }^{b, c 2}$ \\ ${ }^{a}$ APC (Astroparticules et Cosmologie), \\ UMR 7164 (CNRS, Université Paris 7, CEA, Observatoire de Paris) \\ 10, rue Alice Domon et Léonie Duquet, 75205 Paris Cedex 13, France \\ ${ }^{b}$ Institut für Teoretische Physik, Universität Heidelberg, Philosophenweg 16 and 19, \\ D-69120 Heidelberg, Germany \\ ${ }^{c}$ Instituto de Fisica Teorica, UAM/CSIS Facultad de Ciencias C-XVI, \\ C.U. Cantoblanco, E-28049-Madrid, Spain
}

\begin{abstract}
We adopt a covariant formalism to derive exact evolution equations for nonlinear perturbations, in a universe dominated by two scalar fields. These scalar fields are characterized by non-canonical kinetic terms and an arbitrary field space metric, a situation typically encountered in inflationary models inspired by string theory. We decompose the nonlinear scalar perturbations into adiabatic and entropy modes, generalizing the definition adopted in the linear theory, and we derive the corresponding exact evolution equations. We also obtain a nonlinear generalization of the curvature perturbation on uniform density hypersurfaces, showing that on large scales it is sourced only by the nonlinear version of the entropy perturbation. We then expand these equations to second order in the perturbations, using a coordinate based formalism. Our results are relatively compact and elegant and enable one to identify the new effects coming from the non-canonical structure of the scalar fields Lagrangian. We also explain how to analyze, in our formalism, the interesting scenario of multifield Dirac-Born-Infeld inflation.
\end{abstract}

\footnotetext{
${ }^{1}$ E-mail: renaux@apc.univ-paris7.fr

${ }^{2}$ E-mail: g.tasinato@thphys.uni-heidelberg.de
} 


\section{Introduction}

Inflation offers a compelling mechanism to produce a spatially flat and approximately homogeneous universe. Moreover, it also provides a source for small primordial fluctuations that seed the observed large scale structures. Simple models of inflation are realized in terms of a single, slowly rolling scalar field characterized by canonical kinetic terms and a flat potential. In this case, the spectrum of fluctuations is characterized by adiabatic, almost scale invariant density perturbations, with nearly Gaussian distribution on super-horizon scales.

While present-day observations are consistent with these models, there are good theoretical reasons to go beyond the simple hypotheses on which they are based. As an important example, string theory motivates frameworks in which many scalar fields, with non-canonical kinetic terms and not necessarily flat potential, play a role during inflation. Indeed, string theory predicts the existence of a large number of scalar fields, the moduli, which in the early universe may be sufficiently light to interact with the inflaton field, playing an active role during inflation. Moreover, the most studied stringy inflationary models are based on the dynamics of D-branes moving in higher dimensional spaces: in these set-ups, the inflaton field is governed by a DiracBorn-Infeld (DBI) action characterized by non-canonical kinetic terms (see [1] for recent reviews). In some regimes, the non-canonical kinetic terms allow one to obtain inflation also with steep scalar potentials, as in the DBI-inflationary models of [2]-[5]. A more general analysis of the spectrum of primordial fluctuations for multi-field models with non-canonical kinetic terms, then, may allow one to understand how future observations will be able to probe, or exclude, inflationary models motivated or inspired by string theory.

Single-field inflationary models with non-canonical kinetic terms, of a class belonging to models of $k$-inflation [6], have received much attention in the past years, for the possibility to generate spectra of fluctuations with nonGaussian features, at a level that can be probed by future observations (see [7] for review and a comprehensive analysis of this case). More recently, models of inflation with more than one scalar field with non-canonical kinetic terms have been also considered [8]-[16]. In this case, non-standard correlations between adiabatic and entropy modes can occur, and this affects the amplitude of non-Gaussianities in these models [13]-[15]. These correlations can even persist in the primordial radiation dominated era, as first pointed out in [17]. The analysis of the examples studied so far suggests that the dynamics of fluctuations, in string inspired multi-field inflationary models, have new and rich features, depending on the form of kinetic terms for the inflaton field(s).

In this paper, motivated by the previous arguments, we analyze cosmological fluctuations of a system of two scalar fields with non-canonical kinetic terms at a fully nonlinear level. We adopt the covariant formalism developed in [18]-[21], along the lines of earlier works by Ellis and Bruni [22] and Hawking [23] (see also [24]). This formalism is particularly suitable to analyze fluctuations of the system we consider, since it leads to a clear and natural decomposition of fluctuations in adiabatic and entropy components. This separation has been first studied, at the linear level, in [25] for the case of canonical kinetic terms (see [26] for a recent review). It is particularly convenient for analyzing the generation and conversion of adiabatic and isocurvature components of fluctuations produced during inflation. It also plays an important role in the previously mentioned recent papers, that analyze fluctuations for multi-field models with non-canonical kinetic terms.

Following [21], we generalize this decomposition into adiabatic and isocurvature components to a fully nonlinear set-up. In order to do so, we first define adiabatic and isocurvature covectors, representing the nonlinear generalization of linear fluctuations, in the case of general field space metric. Then, we obtain a set of exact evolution equations for these quantities, in our framework characterised by a non-standard Lagrangian. In addition, by defining a suitable nonlinear generalization of the curvature perturbation, we derive the evolution equation for this quantity and we determine how it is sourced by the nonlinear entropy covector. This result generalizes already known linear equations that play an important role for analyzing how entropy fluctuations are converted into adiabatic modes on superhorizon scales.

We then show how our results can be reexpressed in a more familiar coordinate based approach, and how our equations can be expanded to first and second order. Proceeding in this way, we re-obtain at first order the linear evolution equations determined in [11], while we find new results when pursuing the expansion up to second order. In particular, working in a large scale limit, we determine how the curvature perturbation is sourced by the entropy modes at second order. Our final equations are relatively compact, and allow one to clearly appreciate the impact of non-canonical kinetic terms on the evolution of fluctuations.

Our discussion closely follows [21], to which we refer the reader for further details and references. We show how to extend the methods of this paper to the case of non-canonical kinetic terms for the scalar fields, and for general field space metric. In doing so, we obtain nonlinear equations for the fluctuations, that can be 
expressed in a physically transparent way, and we show the usefulness of the covariant approach for analyzing cosmological models inspired by string theory.

\section{Covariant formalism for scalars with non-canonical kinetic terms}

We consider an arbitrary unit timelike vector $u^{a}=d x^{a} / d \tau\left(u_{a} u^{a}=-1\right)$, defining a congruence of cosmological observers. The spatial projection tensor orthogonal to the four-velocity $u^{a}$ is provided by

$$
h_{a b} \equiv g_{a b}+u_{a} u_{b}, \quad\left(h_{b}^{a} h_{c}^{b}=h_{c}^{a}, \quad h_{a}^{b} u_{b}=0\right) .
$$

To describe the time evolution, the covariant definition of the time derivative will be the Lie derivative with respect to $u^{a}$, defined for a generic covector $Y_{a}$ by (see e.g. [27])

$$
\dot{Y}_{a} \equiv \mathcal{L}_{u} Y_{a} \equiv u^{b} \nabla_{b} Y_{a}+Y_{b} \nabla_{a} u^{b},
$$

and will be denoted by a dot. For scalar quantities, one simply has

$$
\dot{f}=u^{b} \nabla_{b} f .
$$

To describe perturbations in the covariant approach, we consider the projected covariant derivative orthogonal to the four-velocity $u^{a}$, denoted by $D_{a}$. For a generic tensor, the definition is

$$
D_{a} T_{b \ldots}^{c \ldots} \equiv h_{a}^{d} h_{b}^{e} \ldots h_{f}^{c} \ldots \nabla_{d} T_{e \ldots}^{f \ldots} .
$$

In particular, when focussing on a scalar quantity $f$, this reduces to

$$
D_{a} f \equiv h_{a}^{b} \nabla_{b} f=\partial_{a} f+u_{a} \dot{f} .
$$

We can also decompose

$$
\nabla_{b} u_{a}=\sigma_{a b}+\omega_{a b}+\frac{1}{3} \Theta h_{a b}-a_{a} u_{b},
$$

with the (symmetric) shear tensor $\sigma_{a b}$ and the (antisymmetric) vorticity tensor $\omega_{a b}$; the volume expansion, $\Theta$, is defined by

$$
\Theta \equiv \nabla_{a} u^{a}
$$

while

$$
a^{a} \equiv u^{b} \nabla_{b} u^{a}
$$

is the acceleration vector.

Let us now consider N scalar fields minimally coupled to gravity with general Lagrangian density (see [11] for a study of the linear perturbations in this type of model):

$$
\mathcal{L}=P\left(X, \phi^{I}\right) .
$$

Here $X$ is

$$
X=-\frac{1}{2} G_{I J} g^{a b} \nabla_{a} \phi^{I} \nabla_{b} \phi^{J},
$$

or, using Eq. (5),

$$
X=\frac{1}{2} G_{I J} \dot{\phi}^{I} \dot{\phi}^{J}-\frac{1}{2} G_{I J} D_{a} \phi^{I} D^{a} \phi^{J}
$$

and $G_{I J} \equiv G_{I J}\left(\phi^{K}\right)$ is a metric in 'field space' that can be used to raise and lower field indices, denoted by capital letters. One can consider more general Lagrangians, like the ones studied in [13, 15].

However, the choice of Lagrangian density Eq. (9) is suitable for describing most of $k$-inflationary models considered in the literature. Especially, it is important to point out that our formalism can be applied to two-field DBI inflationary models of the type studied in $[12,13]$. One of the present author (S. RP) has shown 
in these works that the multi-field DBI Lagrangian can not be written in the form $P\left(X, \phi^{I}\right)$. It can however be written in the form $P\left(\tilde{X}, \phi^{I}\right)$ where $X$ and $\tilde{X}$ only differs by terms in spatial gradients. Therefore, all the results derived in this paper in the large scale limit, where spatial gradients can be neglected, are readily applicable to multi-field DBI models simply by considering the specific Lagrangian

$$
P\left(X, \phi^{I}\right)=-\frac{1}{f\left(\phi^{I}\right)}\left(\sqrt{1-2 f\left(\phi^{I}\right) X}-1\right)-V\left(\phi^{I}\right)
$$

where $f$ is the so-called warp factor and $V$ is a potential term (see [13] for more details on multi-field DBI models).

The energy momentum tensor derived from Eq. (9) reads

$$
T_{a b}=P_{, X} G_{I J} \nabla_{a} \phi^{I} \nabla_{b} \phi^{J}+g_{a b} P .
$$

Given an arbitrary unit timelike vector field $u^{a}$, it is always possible to decompose the total energy momentum tensor as

$$
T_{a b}=(\rho+p) u_{a} u_{b}+q_{a} u_{b}+u_{a} q_{b}+g_{a b} p+\pi_{a b},
$$

where $\rho, p, q_{a}$ and $\pi_{a b}$ are respectively the energy density, pressure, momentum and anisotropic stress tensor measured in the frame defined by $u^{a}$. Starting from the expression for the energy-momentum tensor (13) one finds (we write $\dot{\phi}_{I} \equiv G_{I J} \dot{\phi}^{J}$ )

$$
\begin{aligned}
\rho & \equiv T_{a b} u^{a} u^{b}=P_{, X} \dot{\phi}^{I} \dot{\phi}_{I}-P \\
p & \equiv \frac{1}{3} h^{a c} T_{a b} h_{c}^{b}=\frac{1}{3} P_{,_{X}} G_{I J} D_{a} \phi^{I} D^{a} \phi^{J}+P \\
q_{a} & \equiv-u^{b} T_{b c} h_{a}^{c}=-P_{, X} \dot{\phi}_{I} D_{a} \phi^{I}, \\
\pi_{a b} & \equiv h_{a}^{c} T_{c d} h_{b}^{d}-p h_{a b}=P_{, X}\left(G_{I J} D_{a} \phi^{I} D_{b} \phi^{J}-\frac{h_{a b}}{3} G_{I J} D_{c} \phi^{I} D^{c} \phi^{J}\right) .
\end{aligned}
$$

The evolution equations for the scalar fields are obtained from the variation of the action with respect to the fields themselves. In our case one gets

$$
P_{, X} G_{I J} \nabla^{a} \nabla_{a} \phi^{J}+P_{, X} \Gamma_{I J K}\left(\nabla_{a} \phi^{J}\right)\left(\nabla^{a} \phi^{K}\right)+\left(\nabla^{a} P_{, X}\right) G_{I J} \nabla_{a} \phi^{J}+P_{, I}=0
$$

where $\Gamma_{I J K} \equiv G_{I L} \Gamma_{J K}^{L} \equiv \frac{1}{2}\left(G_{I J, K}+G_{I K, J}-G_{J K, I}\right)$ is the Christoffel symbol associated to the metric $G_{I J}$. The previous equation reduces to the usual one, in the case one chooses $P=X-V\left(\phi^{I}\right)$, where $V$ is a potential.

Notice that using the equalities, following from (5) and (6),

$$
\begin{gathered}
D_{a} D^{a} \phi^{I}=\nabla_{a} \nabla^{a} \phi^{I}+\ddot{\phi}^{I}+\Theta \dot{\phi}^{I}-a^{b} D_{b} \phi^{I}, \\
\nabla^{a} P_{, X} \nabla_{a} \phi^{J}=D^{a} P_{, X} D_{a} \phi^{J}-\dot{P}_{, X} \dot{\phi}^{J}, \\
\nabla^{a} \phi^{J} \nabla_{a} \phi^{K}=D^{a} \phi^{J} D_{a} \phi^{K}-\dot{\phi}^{J} \dot{\phi}^{K},
\end{gathered}
$$

equation (19) becomes

$\ddot{\phi}^{I}+\Gamma_{J K}^{I}\left(\dot{\phi}^{J} \dot{\phi}^{K}-D_{a} \phi^{J} D^{a} \phi^{K}\right)+\left(\Theta+\frac{\dot{P}_{, X}}{P_{, X}}\right) \dot{\phi}^{I}-\frac{1}{P_{, X}} G^{I J} P_{, J}-D_{a} D^{a} \phi^{I}-a^{b} D_{b} \phi^{I}-\frac{\left(D^{b} P_{, X}\right)}{P_{, X}} D_{b} \phi^{I}=0$.

To simplify the notation, it is useful to define the spacetime derivative of field space vectors in curved coordinates

$$
\mathcal{D}_{a} A^{I} \equiv \nabla_{a} A^{I}+\Gamma_{J K}^{I} \nabla_{a} \phi^{J} A^{K},
$$

with which we define a time derivative in field space

$$
\mathcal{D}_{u} A^{I} \equiv u^{a} \mathcal{D}_{a} A^{I}
$$


and a spatially projected derivative in field space

$$
\mathcal{D}_{\perp a} T_{b \ldots}^{I c \ldots} \equiv h_{a}{ }^{d} h_{b}{ }^{e} \ldots h_{f}^{c} \ldots \mathcal{D}_{d} T_{e \ldots}^{I f \ldots} .
$$

Notice that $\mathcal{D}_{a}$ acts as an ordinary time derivative on field space scalars (i.e. quantities without field space indices) and $\mathcal{D}_{a} G_{I J}=0$. Using these definitions, it is possible to rewrite (23) in a more condensed form as

$$
\mathcal{D}_{u} \dot{\phi}^{I}+\left(\Theta+\frac{\dot{P}_{, X}}{P_{, X}}\right) \dot{\phi}^{I}-\frac{1}{P_{, X}} G^{I J} P_{, J}-\mathcal{D}_{\perp a}\left(D^{a} \phi^{I}\right)-a^{b} D_{b} \phi^{I}-\frac{\left(D^{b} P_{, X}\right)}{P_{, X}} D_{b} \phi^{I}=0 .
$$

\section{Single field}

We start our discussion examining the simple situation of a single field. This provides the opportunity to introduce some quantities that will play an important role in the following discussion.

Let us denote by $\phi$ the single scalar field we are considering and set the trivial field space metric $G_{11}=1$. Then the Klein-Gordon equation (27) reads

$$
\ddot{\phi}+\left(\Theta+\frac{\dot{P}_{, X}}{P_{, X}}\right) \dot{\phi}-\frac{1}{P_{, X}} \frac{d P}{d \phi}-D_{a} D^{a} \phi-a^{b} D_{b} \phi-\frac{\left(D^{b} P_{, X}\right)}{P_{, X}} D_{b} \phi=0 .
$$

By choosing

$$
u_{\text {com }}^{a}= \pm \frac{\nabla^{a} \phi}{\sqrt{-\nabla_{a} \phi \nabla^{a} \phi}}
$$

one finds $D_{a} \phi=0$. Then (28) becomes formally identical to the well known homogeneous version, although it remains fully inhomogeneous and nonlinear.

One can proceed further, and derive an exact and covariant equation that mimics the equation of motion governing the linear perturbations of a scalar field in a perturbed FLRW (Friedmann-Lemaitre-RobertsonWalker) spacetime. The idea is to consider the evolution of the space-time gradient of the scalar field, i.e., the covector

$$
\phi_{a} \equiv \nabla_{a} \phi .
$$

Indeed this quantity can be decomposed into a spatial gradient and a longitudinal component,

$$
\phi_{a}=-\dot{\phi} u_{a}+D_{a} \phi .
$$

resembling, within our full nonlinear setting, the usual decomposition of a quantity in a homogeneous part, plus a perturbation. Notice that when making the particular choice (29) the spatial gradient disappears in the above expression, and indeed the evolution equation becomes in this case formally identical to the homogeneous one.

We then construct a second-order (in time) evolution equation for $\phi_{a}$. We recall that a dot stands for the Lie derivative along $u^{a}$, as defined in Eq. (2). We can derive the evolution equation for $\phi_{a}$ by taking the spacetime gradient of Eq. (28) and noting that, for any scalar $\phi$, the Lie derivative with respect to $u^{a}$ and the spacetime gradient (but not the spatial gradient) commute, i.e.,

$$
\nabla_{a} \dot{\phi}=\left(\nabla_{a} \phi\right) .
$$

Acting with the space-time gradient $\nabla_{a}$ on equation (28), and using the previous definitions, we obtain

$$
\begin{aligned}
\ddot{\phi}_{a} & +\left(\Theta+\frac{\dot{P}_{, X}}{P_{, X}}\right) \dot{\phi}_{a}+\dot{\phi} \nabla_{a}\left(\frac{\dot{P}_{, X}}{P_{, X}}\right)-\left[\frac{1}{P_{, X}} \frac{d P}{d \phi}\right]_{, X} \nabla_{a} X-\left[\frac{1}{P_{, X}} \frac{d P}{d \phi}\right]_{, \phi} \phi_{a} \\
& =-\dot{\phi} \nabla_{a} \Theta+\nabla_{a}\left(D_{b} D^{b} \phi\right)+\nabla_{a}\left(a^{b} D_{b} \phi\right)+\nabla_{a}\left(\frac{D^{b} P_{, X}}{P_{, X}} D_{b} \phi\right)
\end{aligned}
$$

This equation is similar to the analogous perturbation equation at linear order, but it additionally incorporates the fully nonlinear dynamics of the scalar field perturbation. 


\subsection{Integrated expansion perturbation on comoving slices}

One can define a covariant generalization of the comoving curvature perturbation using appropriate combinations of spatially projected gradients. For a scalar field, a natural choice is the covariant integrated expansion perturbation on comoving hypersurfaces $\mathcal{R}_{a}$, defined as

$$
\mathcal{R}_{a} \equiv-D_{a} \alpha+\frac{\dot{\alpha}}{\dot{\phi}} D_{a} \phi,
$$

where $\alpha$ is the integrated volume expansion along $u^{a}$,

$$
\alpha \equiv \frac{1}{3} \int d \tau \Theta \quad(\Theta=3 \dot{\alpha})
$$

Since $\Theta / 3$ corresponds to the local Hubble parameter, one sees that the quantity $\alpha$ can be interpreted as the number of e-folds measured along the world-line of a cosmological observer with four-velocity $u^{a}$.

When the four-velocity $u^{a}$ is chosen to be comoving with the scalar field, as defined in Eq. (29), then the last term in the definition (34) drops out.

Note a useful property of $\mathcal{R}_{a}$ : one can replace in its definition (34) the spatial gradients $D_{a}$ by partial or covariant derivatives,

$$
\mathcal{R}_{a}=-\nabla_{a} \alpha+\frac{\dot{\alpha}}{\dot{\phi}} \phi_{a} .
$$

The same property applies to the nonlinear generalization of the comoving Sasaki-Mukhanov variable for a scalar field, which can be defined as

$$
Q_{a} \equiv D_{a} \phi-\frac{\dot{\phi}}{\dot{\alpha}} D_{a} \alpha=\frac{\dot{\phi}}{\dot{\alpha}} \mathcal{R}_{a}
$$

\subsection{Integrated expansion perturbation on uniform energy density slices}

Following $[18,19]$, it is also possible to generalize the curvature perturbation on uniform energy density hypersurfaces. The key role is then played by the covector $\zeta_{a}$ defined as

$$
\zeta_{a} \equiv D_{a} \alpha-\frac{\dot{\alpha}}{\dot{\rho}} D_{a} \rho .
$$

For a perfect fluid, the quantity $\zeta_{a}$ satisfies a simple first-order evolution equation

$$
\dot{\zeta}_{a}=\frac{\Theta^{2}}{3 \dot{\rho}} \Gamma_{a}
$$

where

$$
\Gamma_{a} \equiv D_{a} p-\frac{\dot{p}}{\dot{\rho}} D_{a} \rho
$$

is the nonlinear nonadiabatic pressure perturbation ( $p$ is the pressure measured in the frame defined by $u^{a}$, defined in Eq. (16)). For a barotropic fluid, $\Gamma_{a}=0$ and $\zeta_{a}$ is conserved on all scales. The relation (39) for $\zeta_{a}$ can be seen as a generalization of the familiar conservation law for $\zeta$, the linear curvature perturbation on uniform energy hypersurfaces [28]. In the next sections, we will analyze the form of $\Gamma_{a}$ in various physically interesting situations.

For a single scalar field, the comoving and uniform density integrated expansion perturbations $\zeta_{a}$ and $\mathcal{R}_{a}$ satisfy

$$
\zeta_{a}+\mathcal{R}_{a}=-\frac{\dot{\alpha}}{\dot{\rho}}\left(D_{a} \rho-\frac{\dot{\rho}}{\dot{\phi}} D_{a} \phi\right),
$$

which simply follows from their respective definitions. The right hand side can be interpreted as the "shift" between hypersurfaces of constant $\rho$ and hypersurfaces of constant $\phi$ and the term inside the parenthesis represents the nonlinear generalization of the so-called comoving energy density perturbation of a single scalar field. 
By choosing $u^{a}=u_{\text {com }}^{a}$ as defined in Eq. (29), the energy-momentum tensor of a single scalar field can be written in the perfect fluid form, i.e., with vanishing $q_{a}$ and $\pi_{a b}$ and the energy density $\rho$ and pressure $p$ given by

$$
\rho=P_{, X} \dot{\phi}^{2}-P, \quad p=P,
$$

as can be checked by specializing Eqs. (15-18) to a single field and setting $D_{a} \phi=0$.

After substitution in the definition (40) of the nonadiabatic pressure covector $\Gamma_{a}$, we have

$$
\Gamma_{a}=\frac{\dot{\phi}^{2}}{\dot{\rho}} P_{, X}\left(-\left(1+\frac{1}{c_{s}^{2}}\right) P_{, \phi}+P_{, X \phi} \dot{\phi}^{2}\right) D_{a} \dot{\phi}
$$

where we are using $D_{a} \phi=0$ (implying also $2 X=\dot{\phi}^{2}$ ). We also used

$$
c_{s}^{2}=\frac{p_{, X}}{\rho_{, X}}=\frac{P_{, X}}{P_{, X}+\dot{\phi}^{2} P_{, X X}} .
$$

Then, the evolution equation of $\zeta_{a}$ for a single scalar field is given by

$$
\dot{\zeta}_{a}=\frac{D_{a} \dot{\phi}}{3 P_{, X} \dot{\phi}^{2}}\left[-\left(1+\frac{1}{c_{s}^{2}}\right) P_{, \phi}+P_{, X \phi} \dot{\phi}^{2}\right]
$$

where we have used $\dot{\rho}=-\Theta P_{, X} \dot{\phi}^{2}$ (that can be obtain from the equation of motion for $\phi$ ) to get this expression.

We will show in Sec. 5.2, as a particular case of a more general discussion, that the quantity on the right hand side of equation (45) vanishes on large scales, so that $\zeta_{a}$ is conserved in this limit.

\section{The two-field case}

\subsection{Definition of adiabatic and entropy covectors}

In the two-field case, it is convenient to introduce a particular basis in the field space in which various field dependent quantities are decomposed into adiabatic and entropy components. In the linear theory, this decomposition was first introduced in [25] for two fields. For the multi-field case, it is discussed in [29] in the linear theory (see also [30]) and in [31] in the nonlinear context.

In our case, the corresponding basis consists, in the two-dimensional field space, of a unit vector $\mathrm{e}_{\sigma}^{I}$ defined in the direction of the velocity of the two fields, and thus tangent to the trajectory in field space, and of a unit vector $\mathrm{e}_{s}^{I}$ defined along the direction orthogonal to it (with respect to the field space metric), namely

$$
\mathrm{e}_{\sigma}^{I} \equiv \frac{\dot{\phi}^{I}}{\dot{\sigma}}, \quad G_{I J} \mathrm{e}_{s}^{I} \mathrm{e}_{s}^{J}=1, \quad G_{I J} \mathrm{e}_{s}^{I} \mathrm{e}_{\sigma}^{J}=0,
$$

with

$$
\dot{\sigma} \equiv \sqrt{G_{I J} \dot{\phi}^{I} \dot{\phi}^{J}}
$$

Notice that generically, the quantity $\dot{\sigma}$ is not the derivative along $u^{a}$ of a scalar field $\sigma$; it is merely a notation. An important consequence of the above definitions is the identity

$$
\delta_{J}^{I}=\mathrm{e}_{\sigma}^{I} \mathrm{e}_{\sigma J}+\mathrm{e}_{s}^{I} \mathrm{e}_{s} .
$$

From $e_{\sigma I} e_{\sigma}^{I}=1$, one deduces that $\mathcal{D}_{u} \mathrm{e}_{\sigma}^{I}$ is proportional to $e_{s}^{I}$. It is then convenient to define $\dot{\theta}$ by

$$
\mathcal{D}_{u} \mathrm{e}_{\sigma}^{I}=\dot{\theta} \mathrm{e}_{s}^{I}, \quad \mathcal{D}_{u} \mathrm{e}_{s}^{I}=-\dot{\theta} \mathrm{e}_{\sigma}^{I} .
$$

Again, it is simply a short hand notation; $\dot{\theta}$ is not the derivative along $u^{a}$ of an angle $\theta$, although such an angle can be defined if the field space metric is trivial [21].

Making use of the basis (46), one can then introduce two linear combinations of the scalar field gradients and thus define two covectors, respectively denoted by $\sigma_{a}$ and $s_{a}$, as

$$
\begin{aligned}
\sigma_{a} & \equiv \mathrm{e}_{\sigma I} \nabla_{a} \phi^{I}, \\
s_{a} & \equiv \mathrm{e}_{s I} \nabla_{a} \phi^{I} .
\end{aligned}
$$


We will call these two covectors the adiabatic and entropy covectors, respectively, by analogy with the similar definitions in the linear context [11]. Whereas the entropy covector $s_{a}$ is orthogonal to the four-velocity $u^{a}$, i.e., $u^{a} s_{a}=0$, this is not the case for $\sigma_{a}$ which contains a 'longitudinal' component: $u^{a} \sigma_{a}=\dot{\sigma}$. It turns out to be useful to introduce the spatially projected version of (50-51),

$$
\sigma_{a}^{\perp} \equiv \mathrm{e}_{\sigma I} D_{a} \phi^{I}=\sigma_{a}+\dot{\sigma} u_{a}, \quad s_{a}^{\perp} \equiv \mathrm{e}_{s I} D_{a} \phi^{I}=s_{a} .
$$

\subsubsection{Adiabatic Klein-Gordon equation}

We start our analysis of the evolution equations considering the adiabatic combination of the Klein-Gordon equations, i.e., the contraction of $(27)$ by $\mathrm{e}_{\sigma I}$. By noting that we can write

$$
\dot{\sigma}=\mathrm{e}_{\sigma I} \dot{\phi}^{I} \quad \ddot{\sigma}=\mathrm{e}_{\sigma I} \mathcal{D}_{u} \dot{\phi}^{I},
$$

and by defining ${ }^{3}$

$$
P_{, \sigma} \equiv \mathrm{e}_{\sigma}^{I} P_{, I}
$$

one obtains

$$
\ddot{\sigma}+\left(\Theta+\frac{\dot{P}_{, X}}{P_{, X}}\right) \dot{\sigma}-\frac{1}{P_{, X}} P_{, \sigma}-\mathrm{e}_{\sigma I} \mathcal{D}_{\perp a}\left(D^{a} \phi^{I}\right)-a^{a} \sigma_{a}^{\perp}-\frac{D^{b} P_{, X}}{P_{, X}} \sigma_{b}^{\perp}=0 .
$$

The fourth term can be rewritten as

$$
\mathrm{e}_{\sigma I} \mathcal{D}_{\perp a}\left(D^{a} \phi^{I}\right)=\mathcal{D}_{\perp a}\left(\mathrm{e}_{\sigma I} D^{a} \phi^{I}\right)-\left(\mathcal{D}_{\perp a} \mathrm{e}_{\sigma I}\right) D^{a} \phi^{I}=D^{a} \sigma_{a}^{\perp}-\left(\mathrm{e}_{s I} \mathcal{D}_{\perp a} \mathrm{e}_{\sigma}^{I}\right) s^{a},
$$

where we have used the definition of $\sigma_{a}^{\perp}$, Eq. (52), and the identity (48). Inserting

$$
\mathcal{D}_{a} \dot{\phi}^{I}=u^{b} \mathcal{D}_{b}\left(\nabla_{a} \phi^{I}\right)+\left(\nabla_{a} u^{b}\right)\left(\nabla_{b} \phi^{I}\right)
$$

into the calculation of the Lie derivative of $s_{a}$ with respect to $u_{a}$, one gets

$$
\mathrm{e}_{s I} \mathcal{D}_{a} \mathrm{e}_{\sigma}^{I}=-\mathrm{e}_{\sigma I} \mathcal{D}_{a} \mathrm{e}_{s}^{I}=\frac{1}{\dot{\sigma}}\left(\dot{s}_{a}+\dot{\theta} \sigma_{a}\right),
$$

from which one obtains

$$
\mathrm{e}_{\sigma I} \mathcal{D}_{\perp a}\left(D^{a} \phi^{I}\right)=D^{a} \sigma_{a}^{\perp}-\frac{1}{\dot{\sigma}}\left(\dot{s}_{a}+\dot{\theta} \sigma_{a}^{\perp}\right) s^{a} .
$$

The adiabatic combination of the Klein-Gordon equations can thus be written as

$$
\ddot{\sigma}+\left(\Theta+\frac{\dot{P}_{, X}}{P_{, X}}\right) \dot{\sigma}-\frac{P_{, \sigma}}{P_{, X}}=\nabla^{a} \sigma_{a}^{\perp}-Y_{(s)},
$$

where we have defined

$$
Y_{(s)} \equiv \frac{1}{\dot{\sigma}}\left(\dot{s}_{a}+\dot{\theta} \sigma_{a}^{\perp}\right) s^{a}-\frac{D^{a} P_{, X}}{P_{, X}} \sigma_{a}^{\perp}
$$

and used the property

$$
D^{a} \sigma_{a}^{\perp}+a^{a} \sigma_{a}^{\perp}=\nabla^{a} \sigma_{a}^{\perp}
$$

which is valid for any covector orthogonal to $u^{a}$ (and thus also for $s_{a}$ ). Note that our $Y_{(s)}$ coincides with the one of [21] in the case of canonical kinetic terms, in which situation the last term in (61) vanishes.

Second derivatives of the scalar fields are hidden in the term $\dot{P}_{, X}$. Explicitely, this gives

$$
\frac{\ddot{\sigma}}{c_{s}^{2}}+\Theta \dot{\sigma}+\frac{P_{, X \sigma}}{P_{, X}} \dot{\sigma}^{2}-\frac{P_{, \sigma}}{P_{, X}}=\nabla^{a} \sigma_{a}^{\perp}-Y_{(s)}+\frac{\dot{\sigma} P_{, X X}}{2 P_{, X}}\left(\sigma_{a}^{\perp} \sigma^{\perp a}+s_{a} s^{a}\right)^{\cdot},
$$

where we have introduced the important, spacetime dependent, quantity

$$
c_{s}^{2} \equiv \frac{P_{, X}}{P_{, X}+\dot{\sigma}^{2} P_{, X X}} .
$$

Notice that $c_{s}$ is a fully nonlinear quantity; this implies for example that one cannot replace $\dot{\sigma}^{2}$ by $2 X$ in its definition, since these quantities differ by terms in spatial gradients (see (11)). At linear order however, $c_{s}$ coincides with the now well known speed of sound of $k$-inflationary models $[6,11]$.

\footnotetext{
${ }^{3}$ Similar notations will be employed in the following, such as $P, X \sigma \equiv e_{\sigma}^{I} P, X I, P, X s \equiv e_{s}^{I} P, X I \ldots$
} 


\subsubsection{Entropic Klein-Gordon equation}

Let us now consider the entropic combination of the Klein-Gordon equations, i.e., the contraction of (23) with $\mathrm{e}_{s I}$. By using

$$
\mathrm{e}_{s I} \mathcal{D}_{u} \dot{\phi}^{I}=\dot{\theta} \dot{\sigma}
$$

and by defining the entropic gradient of the potential,

$$
P_{, s} \equiv \mathrm{e}_{s}^{I} P_{, I},
$$

one finds

$$
P_{,_{X}} \dot{\sigma} \dot{\theta}-P_{, s}-P_{,_{X}} \mathrm{e}_{s I} \mathcal{D}_{\perp a}\left(D^{a} \phi^{I}\right)-P_{, X} a^{a} s_{a}-D^{b} P_{,_{X}} s_{b}=0 .
$$

One can rewrite the last three terms by using the identity

$$
\mathrm{e}_{s I} \mathcal{D}_{\perp a}\left(D^{a} \phi^{I}\right)=D^{a} s_{a}+\frac{1}{\dot{\sigma}}\left(\dot{s}_{a}+\dot{\theta} \sigma_{a}^{\perp}\right) \sigma^{\perp a},
$$

derived by inserting (57) into the calculation of the Lie derivative of $\sigma_{a}$ with respect to $u_{a}$. Applying the property (62) to the covector $s_{a}$, one finally gets

$$
\dot{\sigma} \dot{\theta}-\frac{P_{, s}}{P_{, X}}=\nabla_{a} s^{a}+Y_{(\sigma)},
$$

with

$$
Y_{(\sigma)} \equiv \frac{1}{\dot{\sigma}}\left(\dot{s}_{a}+\dot{\theta} \sigma_{a}^{\perp}\right) \sigma^{\perp a}+\frac{D^{a} P_{, X}}{P_{, X}} s_{a}
$$

To conclude, we have shown how to replace the Klein-Gordon equations for the fields by two new equations describing the evolution along the adiabatic and entropy directions respectively. Our covariant equations (63) and (69) look very similar to the homogeneous equations: on the other hand they capture the fully nonlinear dynamics of the scalar fields. Whereas these equations generalize the background evolution equations, we will proceed in the next subsection to derive fully nonlinear and exact equations for the covectors, which mimic and generalize the linearized equations for the adiabatic and entropy components.

\subsection{Evolution of the covectors}

We now derive evolution equations for the covectors $\sigma_{a}$ and $s_{a}$. More precisely, our purpose is to find two evolution equations, which are second order in time (with respect to the Lie derivative along $u^{a}$ ) and which mimic the equations obtained in the linear theory for the perturbations $\delta \sigma$ and $\delta s$ (see [11]).

\subsubsection{Evolution equation for the adiabatic covector}

Starting from the definition of $\sigma_{a}$ in (50), one finds that its time derivative, i.e., the Lie derivative with respect to $u^{a}$, is given by

$$
\dot{\sigma}_{a}=\mathrm{e}_{\sigma I} \mathcal{D}_{a} \dot{\phi}^{I}+\dot{\theta} s_{a}=\nabla_{a} \dot{\sigma}+\dot{\theta} s_{a},
$$

where the last equality is obtained by using $\dot{\phi}^{I}=\dot{\sigma} \mathrm{e}_{\sigma}^{I}$. A further time derivative yields

$$
\ddot{\sigma}_{a}=\nabla_{a} \ddot{\sigma}+\ddot{\theta} s_{a}+\dot{\theta} \dot{s}_{a}
$$

The next step consists in using (60) to eliminate $\ddot{\sigma}$ in the above expression. This gives

$$
\begin{aligned}
\ddot{\sigma}_{a} & +\Theta \dot{\sigma}_{a}+\dot{\sigma} \nabla_{a}\left(\Theta+\frac{\dot{P}_{, X}}{P_{, X}}\right)+\frac{\dot{P}_{, X} \dot{\sigma}_{a}}{P_{, X}}+\frac{P_{, \sigma} \nabla_{a} P_{, X}}{P_{, X}^{2}}-\left(\frac{P_{, \sigma \sigma}}{P_{, X}}+\dot{\theta} \frac{P_{, s}}{P_{, X} \dot{\sigma}}\right) \sigma_{a}-\nabla_{a}\left(\nabla^{c} \sigma_{c}^{\perp}\right)-\frac{P_{, X \sigma} \nabla_{a} X}{P_{, X}} \\
& =\left(\dot{\theta}+\frac{P_{, s}}{\dot{\sigma} P_{, X}}\right) \dot{s}_{a}+\left[\ddot{\theta}+\frac{P_{, \sigma s}}{P_{, X}}+\dot{\theta}\left(\Theta+\frac{\dot{P}_{, X}}{P_{, X}}\right)\right] s_{a}-\nabla_{a} Y_{(s)},
\end{aligned}
$$


where we have used the relation

$$
\nabla_{a} P_{, \sigma}=P_{, \sigma \sigma} \sigma_{a}+P_{, \sigma s} s_{a}+\frac{P_{, s}}{\dot{\sigma}}\left(\dot{s}_{a}+\dot{\theta} \sigma_{a}\right)+P_{, X \sigma} \nabla_{a} X,
$$

and introduced the notation

$$
P_{, \sigma \sigma} \equiv \mathrm{e}_{\sigma}^{I} \mathrm{e}_{\sigma}^{J} \mathcal{D}_{I} \mathcal{D}_{J} P, \quad P_{, s s} \equiv \mathrm{e}_{s}^{I} \mathrm{e}_{s}^{J} \mathcal{D}_{I} \mathcal{D}_{J} P, \quad P_{, s \sigma} \equiv \mathrm{e}_{s}^{I} \mathrm{e}_{\sigma}^{J} \mathcal{D}_{I} \mathcal{D}_{J} P
$$

In the previous expressions, $\mathcal{D}_{I}$ denotes the covariant derivative associated with $G_{I J}$ (thus having $\mathcal{D}_{I} \mathcal{D}_{J} P \equiv$ $\left.P_{, I J}-\Gamma_{I J}^{K} P_{, K}\right)$.

The evolution equation for $\sigma_{a}$ can then be decomposed into a longitudinal part, obtained by contracting (73) with $u^{a}$, and an orthogonal part obtained by contraction with $h_{a b}$. By using the relation

$$
\dot{P}_{, \sigma}=P_{, \sigma \sigma} \dot{\sigma}+\dot{\theta} P_{, s}+P_{, X \sigma} \dot{X},
$$

it is not difficult to see that the longitudinal part yields in fact the time derivative of (63). What is more interesting is the orthogonal or spatial part. Using several equations collected in the appendix, one can expand the various quantities inside the previous expression in terms of the perturbations $\sigma_{a}^{\perp}$ and $s_{a}$. After a long but straightforward calculation, the evolution equation for the adiabatic covector can be written in a form close its linear counterpart [11]:

$$
\begin{aligned}
& \left(\ddot{\sigma}_{a}\right)^{\perp}+\left[\Theta+\frac{c_{s}^{2}}{P_{, X}}\left(\frac{P_{, X}}{c_{s}^{2}}\right)^{\cdot}+\left(c_{s}^{2}-1\right) \frac{Z_{(s)}}{\dot{\sigma}}\right]\left(\dot{\sigma}_{a}\right)^{\perp}+\frac{c_{s}^{2}}{P_{, X}}\left[-P_{, \sigma \sigma}-\frac{\dot{\theta} P_{, s}}{\dot{\sigma}}+e^{-3 \alpha}\left(e^{3 \alpha} \dot{\sigma} P_{, X \sigma}\right)^{\cdot}-P_{, X \sigma} Z_{(s)}\right] \sigma_{a}^{\perp} \\
& +\dot{\sigma} c_{s}^{2} D_{a} \Theta-c_{s}^{2} D_{a}\left(\nabla^{c} \sigma_{c}^{\perp}\right)=\left[\Xi+\frac{Z_{(\sigma)}}{\dot{\sigma}}\right] \dot{s}_{a}+\left[\dot{\Xi}-\Xi\left(2 \frac{\dot{c}_{s}}{c_{s}}-\frac{P_{, \sigma}}{P_{, X} \dot{\sigma}}\right)+M\right] s_{a} \\
& \quad-c_{s}^{2} D_{a} Y_{(s)}+\frac{1}{2 \dot{\sigma}}\left(1-c_{s}^{2}\right) D_{a} \dot{\Pi}+\frac{c_{s}^{2}}{2 P_{, X}}\left[P_{, X \sigma}-P_{, X X} Z_{(s)}+e^{-3 \alpha}\left(e^{3 \alpha} \dot{\sigma} P_{, X X}\right)^{\cdot}\right] D_{a} \Pi
\end{aligned}
$$

In the previous equation we introduced various quantities:

$$
\begin{aligned}
Z_{(\sigma)} & \equiv \nabla_{a} s^{a}+Y_{(\sigma)}, \quad Z_{(s)} \equiv \nabla^{a} \sigma_{a}^{\perp}-Y_{(s)} \\
\Pi & \equiv \sigma_{c}^{\perp} \sigma^{\perp c}+s_{c} s^{c}, \\
\Xi & \equiv \frac{1}{\dot{\sigma} P_{, X}}\left[\left(1+c_{s}^{2}\right) P_{, s}-c_{s}^{2} \dot{\sigma}^{2} P_{, X s}\right]
\end{aligned}
$$

and

$M=\frac{\dot{Z}_{(\sigma)}}{\dot{\sigma}}+\frac{c_{s}^{2} P_{, s} Z_{(s)}}{P_{, X} \dot{\sigma}^{2}}+\frac{c_{s}^{2} Z_{(\sigma)}}{\dot{\sigma}}\left(2 \Theta+\frac{P_{, X \sigma} \dot{\sigma}}{P_{, X}}+\frac{\dot{\sigma} P_{\sigma} P_{, X X}}{P_{, X}^{2}}-2 \frac{\dot{c}_{s}}{c_{s}^{3}}-\frac{P_{, X X} \dot{\Pi}}{P_{, X}}-\frac{Z_{(s)}}{\dot{\sigma}}\right)+\frac{c_{s}^{2} \dot{\Pi}}{2 P_{, X} \dot{\sigma}}\left[P_{, X s}-\frac{P_{, X X}}{P_{, X}} P_{, s}\right]$.

Moreover, we used the integrated volume expansion $\alpha$ introduced in Eq. (35).

Let us make a few comparisions with the case of a standard Lagrangian: the friction term is not only given by the volume expansion $\Theta$ but has a non vanishing contribution coming from the nonlinear speed of sound $c_{s}$, which may vary in time $\left(\dot{c}_{s} \neq 0\right)$ and may be different from unity $\left(c_{s}^{2} \neq 1\right)$. The last two terms in (77), which exhibit a rich dependence on the form of the kinetic term, also vanish for a standard Langrangian $P=X-V\left(\phi^{I}\right)$. Moreover, in that case, $\Xi$ reduces to $2 \dot{\theta}$, which encodes all the multi-field effects at linear order, as shown in [25]. The important parameter $\Xi$ plays an equivalent role for Lagrangians of the form $P\left(X, \phi^{I}\right)$ studied in this paper, as shown in [11]: if $\Xi=0$, the situation is effectively single-field at linear order.

\subsubsection{Evolution equation for the entropy covector}

Let us now consider the evolution equation for $s_{a}$. From Eq. (58) the time derivative of $s_{a}$ is given by

$$
\dot{s}_{a}=\dot{\sigma} \mathrm{e}_{s I} \mathcal{D}_{a} \mathrm{e}_{\sigma}^{I}-\dot{\theta} \sigma_{a} .
$$


Taking another time derivative, one finds

$$
\ddot{s}_{a}=-\ddot{\theta} \sigma_{a}-\dot{\theta} \dot{\sigma}_{a}+\frac{\ddot{\sigma}}{\dot{\sigma}}\left(\dot{s}_{a}+\dot{\theta} \sigma_{a}\right)+\dot{\sigma} \nabla_{a} \dot{\theta}-\dot{\sigma}^{2} R_{K L J}^{I} e_{s I} e_{\sigma}^{J} e_{\sigma}^{K} \nabla_{a} \phi^{L},
$$

where we have used (58) and (49) and the equality

$$
\mathcal{D}_{a}\left(\mathcal{D}_{u} A^{I}\right)-\mathcal{D}_{u}\left(\mathcal{D}_{a} A^{I}\right)=R_{K L J}^{I} \nabla_{a} \phi^{L} \dot{\phi}^{J} A^{K}
$$

applied to the vector $e_{\sigma}^{I}$ and where $R_{K L J}^{I}$ is the Riemann tensor associated to the metric $G_{I J}{ }^{4}$. We can now use the entropic equation (69) to get rid of $\nabla_{a} \dot{\theta}$. Furthermore, using the relation

$$
\nabla_{a} P_{, s}=P_{, s \sigma} \sigma_{a}+P_{, s s} s_{a}-\frac{P_{, \sigma}}{\dot{\sigma}}\left(\dot{s}_{a}+\dot{\theta} \sigma_{a}\right)+P_{, X s} \nabla_{a} X
$$

we obtain

$$
\begin{aligned}
\ddot{s}_{a}- & \frac{1}{\dot{\sigma}}\left(\ddot{\sigma}-\frac{P_{, \sigma}}{P_{, X}}\right) \dot{s}_{a}-\left(\frac{P_{, s s}}{P_{, X}}+\dot{\theta}^{2}\right) s_{a}-\nabla_{a}\left(\nabla_{c} s^{c}\right)-\frac{P_{, X s}}{P_{, X}} \nabla_{a} X+\dot{\sigma}^{2} R_{K L J}^{I} e_{s I} e_{\sigma}^{J} e_{\sigma}^{K} \nabla_{a} \phi^{L} \\
& =-2 \dot{\theta} \dot{\sigma}_{a}-P_{, s} \frac{\nabla_{a} P_{, X}}{P_{, X}^{2}}+\left[\frac{\dot{\theta}}{\dot{\sigma}}\left(\ddot{\sigma}-\frac{P_{, \sigma}}{P_{, X}}\right)-\ddot{\theta}+\frac{P_{, \sigma s}}{P_{, X}}\right] \sigma_{a}+\nabla_{a} Y_{(\sigma)} .
\end{aligned}
$$

As for the adiabatic equation, the longitudinal part of this equation, upon using the relation

$$
\dot{P}_{, s}=P_{, \sigma s} \dot{\sigma}-\dot{\theta} P_{, \sigma}+P_{, X s} \dot{X},
$$

yields the time derivative of Eq. (69). The orthogonal part, instead, yields

$$
\begin{aligned}
\ddot{s}_{a}- & \frac{1}{\dot{\sigma}}\left(\ddot{\sigma}-\frac{P_{, \sigma}}{P_{, X}}\right) \dot{s}_{a}-\left(\frac{P_{, s s}}{P_{, X}}+\dot{\theta}^{2}-\dot{\sigma}^{2} R_{I K L J} e_{s}^{I} e_{s}^{L} e_{\sigma}^{J} e_{\sigma}^{K}\right) s_{a}-D_{a}\left(\nabla_{c} s^{c}\right)-\frac{P_{, X s}}{P_{, X}} D_{a} X \\
& =-2 \dot{\theta}\left(\dot{\sigma}_{a}\right)^{\perp}-P_{, s} \frac{D_{a} P_{, X}}{P_{, X}^{2}}+\left[\frac{\dot{\theta}}{\dot{\sigma}}\left(\ddot{\sigma}-\frac{P_{, \sigma}}{P_{, X}}\right)-\ddot{\theta}+\frac{P_{, \sigma s}}{P_{, X}}\right]\left(\sigma_{a}\right)^{\perp}+D_{a} Y_{(\sigma)},
\end{aligned}
$$

where we have used the property that the covectors $\dot{s}_{a}$ and $\ddot{s}_{a}$ are purely spatial, i.e., that $\left(\dot{s}_{a}\right)^{\perp}=\dot{s}_{a}$ and $\left(\ddot{s}_{a}\right)^{\perp}=\ddot{s}_{a}$. Using (69) as well as various expressions collected in the appendix, we finally obtain

$$
\begin{aligned}
\ddot{s}_{a}+ & \left(\Theta+\frac{\dot{P}_{X}}{P_{, X}}-\frac{Z_{(s)}}{\dot{\sigma}}\right) \dot{s}_{a}+\left(\mu_{s}^{2}-\frac{Z_{(\sigma)}^{2}}{\dot{\sigma}^{2}}-\frac{Z_{(\sigma)} \Xi}{\dot{\sigma} c_{s}^{2}}\right) s_{a}-D_{a}\left(\nabla_{c} s^{c}\right) \\
= & -\left(\frac{\Xi}{c_{s}^{2}}+2 \frac{Z_{(\sigma)}}{\dot{\sigma}}\right)\left(\dot{\sigma}_{a}\right)^{\perp}+\left[\frac{\ddot{\sigma}}{\dot{\sigma}}\left(\frac{\Xi}{c_{s}^{2}}+2 \frac{Z_{(\sigma)}}{\dot{\sigma}}\right)-\frac{\dot{Z}_{(\sigma)}}{\dot{\sigma}}\right]\left(\sigma_{a}\right)^{\perp}+D_{a} Y_{(\sigma)} \\
& -\frac{1}{2 P_{, X}}\left(P_{, X s}-\frac{P_{, X X} P_{, s}}{P_{, X}}\right)\left(D_{a} \Pi-\frac{\dot{\Pi}}{\dot{\sigma}} \sigma_{a}^{\perp}\right)
\end{aligned}
$$

with

$$
\mu_{s}^{2} \equiv-\frac{P_{, s s}}{P_{, X}}+\dot{\sigma}^{2} R_{I K L J} e_{s}^{I} e_{s}^{L} e_{\sigma}^{J} e_{\sigma}^{K}-\frac{P_{, s}^{2}}{c_{s}^{2} \dot{\sigma}^{2} P_{, X}^{2}}+\frac{2 P_{, X s} P_{, s}}{P_{, X}^{2}}
$$

which mimics its linear counterpart in [11]. Let us remark that the last term in (89), absent in the linear theory, also vanishes for a standard Lagrangian.

Starting from the fully nonlinear Klein-Gordon equations, we thus obtained a system of two coupled equations (77) and (89), controlling the evolution of our nonlinear adiabatic and entropy components. Although they appear quite involved, we will learn that they simplify considerably in physically interesting situations, in particular when considering linear or large scale limits. Moreover, as we will see later, immediately deduced from these equations are the already known evolution equations for the linear adiabatic and entropy components. Furthermore, since our equations are exact, they can be used to go beyond the linear order, up to second or higher orders in the expansion.

\footnotetext{
${ }^{4}$ We thus have $R_{K L J}^{I}=\partial_{L} \Gamma_{K J}^{I}-\partial_{J} \Gamma_{K L}^{I}+\Gamma_{L M}^{I} \Gamma_{K J}^{M}-\Gamma_{J M}^{I} \Gamma_{K L}^{M}$.
} 


\subsection{Generalized covariant perturbations}

In this subsection, we will be first interested in the covariant generalization of the comoving energy density and curvature perturbations in the context of a two-field system. We will then consider the generalization of the curvature perturbation on uniform energy density hypersurfaces.

\subsubsection{Comoving energy density covector}

Let us introduce the covector

$$
\epsilon_{a} \equiv D_{a} \rho-\frac{\dot{\rho}}{\dot{\sigma}} \sigma_{a}^{\perp}
$$

which can be interpreted as a covariant generalization of the comoving energy density perturbation. In order to obtain the explicit expression of $\epsilon_{a}$ in terms of $\sigma_{a}$ and $s_{a}$, let us rewrite the components (15-18) of the energy-momentum tensor in the form

$$
\begin{aligned}
\rho & =P_{, X} \dot{\sigma}^{2}-P, \\
p & =\frac{P_{, X}}{3} \Pi+P, \\
q_{a} & =-P_{, X} \dot{\sigma} \sigma_{a}^{\perp}, \\
\pi_{a b} & =P_{, X}\left(\Pi_{a b}-\frac{1}{3} h_{a b} \Pi\right),
\end{aligned}
$$

where we have defined

$$
\Pi_{a b} \equiv \sigma_{a}^{\perp} \sigma_{b}^{\perp}+s_{a} s_{b},
$$

while the quantity $\Pi$ has already been introduced in (79).

Using (92), one finds

$$
\begin{aligned}
D_{a} \rho & =\left(P_{X_{X} X} \dot{\sigma}^{2}-P_{, X}\right)\left(\dot{\sigma} D_{a} \dot{\sigma}-\frac{D_{a} \Pi}{2}\right)+2 P_{, X} \dot{\sigma} D_{a} \dot{\sigma}-P_{, \sigma} \sigma_{a}^{\perp}-P_{, s} s_{a}+\dot{\sigma}^{2}\left(P_{, X \sigma} \sigma_{a}^{\perp}+P_{, X s} s_{a}\right), \\
\dot{\rho} & =\left(P_{, X X} \dot{\sigma}^{2}-P_{, X}\right)\left(\dot{\sigma} \ddot{\sigma}-\frac{\dot{\Pi}}{2}\right)+2 P_{, X} \dot{\sigma} \ddot{\sigma}-P_{, \sigma} \dot{\sigma}+P_{, X \sigma} \dot{\sigma}^{3},
\end{aligned}
$$

from which one obtains

$$
\epsilon_{a}=\frac{P_{, X}}{c_{s}^{2}}\left(\dot{\sigma}\left(\dot{\sigma}_{a}\right)^{\perp}-\ddot{\sigma} \sigma_{a}^{\perp}\right)-\frac{P_{, X}}{c_{s}^{2}}\left(\dot{\sigma} \Xi+Z_{(\sigma)}\right) s_{a}+\frac{1}{2}\left(P_{, X}-\dot{\sigma}^{2} P_{, X X}\right)\left(D_{a} \Pi-\frac{\dot{\Pi}}{\dot{\sigma}} \sigma_{a}^{\perp}\right) .
$$

This expression can be employed to rewrite Eq. (89) for the entropy covector $s_{a}$ in a useful different form. As noticed above, the longitudinal projection of Eq. (86) yields the time derivative of (69), which reads

$$
-\frac{\dot{\theta}}{\dot{\sigma}} \frac{P_{, \sigma}}{P_{, X}}-\ddot{\theta}+\frac{P_{, \sigma s}}{P_{, X}}=\frac{\dot{\theta}}{\dot{\sigma}} \ddot{\sigma}-\frac{P_{, X} \dot{X}}{P_{, X} \dot{\sigma}}+\frac{\dot{P}_{, X} P_{, s}}{\dot{\sigma} P_{, X}^{2}}-\frac{\dot{Z}_{(\sigma)}}{\dot{\sigma}} .
$$

This relation, together with Eqs. (60) and (98), enables us to reexpress (89) as

$$
\begin{aligned}
\ddot{s}_{a}+ & {\left[\Theta+\frac{\dot{P}_{, X}}{P_{, X}}-\frac{Z_{(s)}}{\dot{\sigma}}\right] \dot{s}_{a}+\left[\mu_{s}^{2}+\frac{\Xi^{2}}{c_{s}^{2}}+\frac{Z_{(\sigma)}}{\dot{\sigma}}\left(\frac{Z_{(\sigma)}}{\dot{\sigma}}+2 \Xi\right)\right] s_{a}-D_{a}\left(\nabla_{c} s^{c}\right) } \\
= & -\frac{1}{P_{, X} \dot{\sigma}}\left(\Xi+2 \frac{c_{s}^{2}}{\dot{\sigma}} Z_{(\sigma)}\right) \epsilon_{a}+\frac{1}{\dot{\sigma}}\left(1-\frac{1}{2 c_{s}^{2}}\right)\left(\Xi+2 \frac{c_{s}^{2}}{\dot{\sigma}} Z_{(\sigma)}\right)\left(D_{a} \Pi-\frac{\dot{\Pi}}{\dot{\sigma}} \sigma_{a}^{\perp}\right) \\
& -\frac{\dot{Z}_{(\sigma)}}{\dot{\sigma}} \sigma_{a}^{\perp}+D_{a} Y_{(\sigma)} .
\end{aligned}
$$

As in the linear theory [11], this gives us an alternative expression for the evolution equation for $s_{a}$, in which the comoving energy density perturbation appears explicitely on the right hand side. This expression will be useful in Sec. 5.2 when discussing the large-scale evolution of $s_{a}$. 


\subsubsection{Comoving curvature covector}

Together with the comoving energy density, the comoving curvature perturbation can be generalized. For the general case of several scalar fields, this is done by defining the comoving integrated expansion perturbation (recall the expression for $q_{a}$ in (94)),

$$
\mathcal{R}_{a} \equiv-D_{a} \alpha-\frac{\dot{\alpha}}{P_{, X} \dot{\sigma}^{2}} q_{a} .
$$

The definition of the Sasaki-Mukhanov covector given for a single scalar field in Eq. (37) can then be extended to the case of several fields, by defining for each field

$$
Q_{a}^{I} \equiv D_{a} \phi^{I}-\frac{\dot{\phi}^{I}}{\dot{\alpha}} D_{a} \alpha .
$$

Thus, the comoving covector $\mathcal{R}_{a}$ can also be written as

$$
\mathcal{R}_{a}=\frac{\dot{\alpha}}{\left(\dot{\phi}_{J} \dot{\phi}^{J}\right)} \dot{\phi}_{I} Q_{a}^{I} .
$$

In the two-field case, the definition (101) reduces, using (94), to

$$
\mathcal{R}_{a} \equiv-D_{a} \alpha+\frac{\dot{\alpha}}{\dot{\sigma}} \sigma_{a}^{\perp} .
$$

Furthermore, one can generalize the Sasaki-Mukhanov variable to the adiabatic covector by defining

$$
Q_{a} \equiv \mathrm{e}_{\sigma I} Q_{a}^{I}=\sigma_{a}^{\perp}-\frac{\dot{\sigma}}{\dot{\alpha}} D_{a} \alpha .
$$

Following [21], instead of giving the evolution equation of $\mathcal{R}_{a}$, we will use a fluid description by considering the covariant generalization of the uniform density curvature perturbation, i.e., the integrated expansion perturbation on uniform density hypersurfaces $\zeta_{a}$, defined in Eq. (38). In the two-field case, these two quantities are related by

$$
\zeta_{a}+\mathcal{R}_{a}=-\frac{\dot{\alpha}}{\dot{\rho}} \epsilon_{a} .
$$

In contrast with the case of a single scalar field, which can always be described as a perfect fluid, the total energy-momentum for two or more scalar fields corresponds in general to that of a dissipative fluid: the nonlinear formalism developed in [20] will thus be useful in this case.

The adiabatic Klein-Gordon equation (63) can be rewritten as a continuity equation for the total energy density (92) and pressure (93), which reads

$$
\dot{\rho}+\Theta(\rho+p)=\mathcal{D},
$$

with the dissipative term

$$
\mathcal{D}=P_{, X}\left(\dot{\sigma} Z_{(s)}+\frac{1}{3} \Theta \Pi+\frac{1}{2} \dot{\Pi}\right) .
$$
by

In [20] it was shown that the evolution equation for $\zeta_{a}$ for a dissipative fluid, which generalizes (39), is given

$$
\dot{\zeta}_{a}=\frac{\Theta^{2}}{3 \dot{\rho}}\left(\Gamma_{a}+\Sigma_{a}\right),
$$

where the second source term on the right hand side, due to the dissipative nature of the fluid, is defined in terms of $\mathcal{D}$ as

$$
\Sigma_{a} \equiv-\frac{1}{\Theta}\left(D_{a} \mathcal{D}-\frac{\dot{\mathcal{D}}}{\dot{\rho}} D_{a} \rho\right)+\frac{\mathcal{D}}{\Theta^{2}}\left(D_{a} \Theta-\frac{\dot{\Theta}}{\dot{\rho}} D_{a} \rho\right) .
$$

Let us return to the covector $\Gamma_{a}$. Substituting the expression (93) for $p$ into its definition (40), as well as using

$$
\begin{aligned}
\dot{P} & =P_{, \sigma} \dot{\sigma}+P_{, X}\left(\dot{\sigma} \ddot{\sigma}-\frac{1}{2} \dot{\Pi}\right), \\
D_{a} P & =P_{, \sigma} \sigma_{a}^{\perp}+P_{, s} s_{a}+P_{, X}\left(\dot{\sigma}\left(\dot{\sigma}_{a}^{\perp}-\dot{\theta} s_{a}\right)-\frac{1}{2} D_{a} \Pi\right),
\end{aligned}
$$


one obtains

$$
\Gamma_{a}=-\epsilon_{a} \frac{\dot{\sigma}}{\dot{\rho}}\left[\left(1+c_{s}^{2}\right) P_{, \sigma}-c_{s}^{2} \dot{\sigma}^{2} P_{, X \sigma}\right]+P_{, X} \dot{\sigma} \Xi s_{a}-P_{, X} c_{s}^{2}\left(D_{a} \Pi-\frac{\dot{\Pi}}{\dot{\rho}} D_{a} \rho\right)+\frac{1}{3}\left(D_{a}\left(P_{, X} \Pi\right)-\frac{\left(P_{, X} \Pi\right)^{\cdot}}{\dot{\rho}} D_{a} \rho\right)
$$

where we have introduced $\epsilon_{a}$ defined in Eq. (91). The above equation expresses the nonlinear nonadiabatic pressure perturbation in the two-field case, which sources Eq. (109). It will be useful in the next section, where we will consider both the linear and super-Hubble approximations of our evolution equations.

The evolution of $\zeta_{a}$, governed by Eq. (109), is thus sourced by a rather complicated term obtained by summing $\Gamma_{a}$ and $\Sigma_{a}$ in Eq. (110). We will learn in the next sections that this term simplifies considerably in physically interesting cases, when either the linear or the super-Hubble approximations are taken.

\section{Approximate equations}

In this section we study the evolution equations of the two-field system under two types of approximations: the linear limit and the limit in which we neglect higher orders in spatial gradients. In an expanding FLRW universe, the latter corresponds to the large scale limit. In the rest of the paper we will use the symbol $\simeq$ to denote an equality at the linear level, and the symbol $\approx$ to denote an equality valid only on large scales.

\subsection{Homogeneous and linearized equations}

In many cosmological applications, since our Universe appears to be close to a FLRW universe on large scales, it is sufficient to restrict oneself to the linearized version of the evolution equations. We first consider this linearization procedure directly at the level of the covariant equations, as done in [22].

In a strictly FLRW universe, all the spatial gradients vanish and therefore

$$
\sigma_{a}^{\perp}=0 \quad, \quad s_{a}=0 . \quad(\text { FLRW })
$$

Consequently, the scalar quantities $Y_{(s)}$ and $Y_{(\sigma)}$, defined respectively in (61) and (70), vanish and the evolution equations for $\sigma$ and $\theta$, respectively (60) and (69), reduce to

$$
\begin{array}{rlrl}
\ddot{\sigma}+\left(3 H+\frac{\dot{P}_{, X}}{P_{, X}}\right) \dot{\sigma}-\frac{P_{, \sigma}}{P_{, X}} & =0, & & \text { (FLRW }) \\
\dot{\sigma} \dot{\theta}-\frac{P_{, s}}{P_{, X}} & =0 & \text { (FLRW })
\end{array}
$$

where we have introduced the Hubble parameter $H=\Theta / 3$. Not surprisingly, the above equations exactly correspond to the homogeneous equations given in [11]. Furthermore, in a FLRW universe, all the terms in Eq. (77) for $\sigma_{a}^{\perp}$ and Eq. (100) for $s_{a}$ vanish.

At linearized order, we treat the covectors $\sigma_{a}^{\perp}$ and $s_{a}$, vanishing at zeroth order, as first-order quantities. Similarly, their derivatives $\dot{s}_{a}, \ddot{s}_{a},\left(\dot{\sigma}_{a}\right)^{\perp}$ and $\left(\ddot{\sigma}_{a}\right)^{\perp}$ are first-order quantities. Therefore the linearized version of the evolution equations are simply obtained by keeping only the homogeneous terms in the coefficients multiplying the spatial projection of $\sigma_{a}, s_{a}$ and their derivatives. The linearized versions of (77) and (100) are thus (recall the definition of $\Xi$ in (80))

$$
\begin{aligned}
& \left(\ddot{\sigma}_{a}\right)^{\perp}+\left[3 H+\frac{c_{s}^{2}}{P_{, X}}\left(\frac{P_{, X}}{c_{s}^{2}}\right)^{\cdot}\right]\left(\dot{\sigma}_{a}\right)^{\perp}+\frac{c_{s}^{2}}{P_{, X}}\left[-P_{, \sigma \sigma}-P_{, X} \dot{\theta}^{2}+e^{-3 \alpha}\left(e^{3 \alpha} \dot{\sigma} P_{, X \sigma}\right)^{\cdot}\right] \sigma_{a}^{\perp} \\
& +\dot{\sigma} c_{s}^{2} D_{a} \Theta-c_{s}^{2} D_{a}\left(D^{c} \sigma_{c}^{\perp}\right) \simeq \Xi \dot{s}_{a}+\left[\dot{\Xi}-\Xi\left(\frac{\left(H c_{s}^{2}\right)^{\cdot}}{H c_{s}^{2}}-\frac{P_{, \sigma}}{P_{, X} \dot{\sigma}}-\frac{\dot{H}}{H}\right)\right] s_{a}
\end{aligned}
$$

and

$$
\ddot{s}_{a}+\left(3 H+\frac{\dot{P}_{, X}}{P_{, X}}\right) \dot{s}_{a}+\left(\mu_{s}^{2}+\frac{\Xi^{2}}{c_{s}^{2}}\right) s_{a}-D_{a}\left(D_{c} s^{c}\right) \simeq-\frac{\Xi}{P_{, X} \dot{\sigma}} \epsilon_{a} .
$$


Note that the terms involving $Y_{(s)}, Y_{(\sigma)}$ and $\Pi$ have disappeared, since these scalars are quadratic in first-order quantities. We have also replaced $\nabla^{c} \sigma_{c}^{\perp}$ by $D^{c} \sigma_{c}^{\perp}$, as well as $\nabla^{c} s_{c}$ by $D^{c} s_{c}$, since their difference is quadratic in first-order quantities, according to (62). Indeed, the acceleration vector $a^{b}$, which vanishes at zeroth order, is considered as a first-order quantity.

One can also linearize the evolution equation for $\zeta_{a}$. As discussed above, the terms containing $Y_{(s)}$ and $\Pi$ can be neglected. The dissipative term $\mathcal{D}$ thus reduces to

$$
\mathcal{D} \simeq P_{, X} \dot{\sigma} D^{a} \sigma_{a}^{\perp}
$$

while the expression for $\epsilon_{a}$ becomes

$$
\epsilon_{a} \simeq \frac{P_{, X}}{c_{s}^{2}}\left[\dot{\sigma} \dot{\sigma}_{a}^{\perp}-\ddot{\sigma} \sigma_{a}^{\perp}-\dot{\sigma} \Xi s_{a}\right]
$$

On the other hand, the expression for $\Gamma_{a}$ becomes

$$
\Gamma_{a} \simeq-\epsilon_{a} \frac{\dot{\sigma}}{\dot{\rho}}\left[\left(1+c_{s}^{2}\right) P_{, \sigma}-c_{s}^{2} \dot{\sigma}^{2} P_{, X \sigma}\right]+P_{, X} \dot{\sigma} \Xi s_{a},
$$

from which it is immediate to obtain the expression for $\dot{\zeta}_{a}$.

\subsection{Expansion in spatial gradients}

Apart from the linearization procedure, there is another approximation in the cosmological context that is used to describe the Universe on very large scales. This approximation is based on an expansion in spatial gradients, which are small for scales larger than the local Hubble radius [32, 33, 34]. Recently for instance, it has been adopted in [35] for studying nonlinear perturbations by means of the ADM formalism, in a system describing a single scalar field with arbitrary kinetic terms, up to second order in the gradient expansion. In the present article, we restrict ourselves to the leading order: in this perspective one sees, from their definition (52), that $\sigma_{a}^{\perp}$ and $s_{a}$ are first-order quantities with respect to spatial gradients because they are linear combinations of spatial gradients. The scalars $Y_{(s)}$ and $Y_{(\sigma)}$ however are second-order with respect to spatial gradients since they are quadratic in $\sigma_{a}^{\perp}$ and $s_{a}$ (or their time derivatives). Hence, the right hand side of Eq. (60) and of Eq. (69) can be neglected on large scales, so that these two equations become, in the large-scale limit,

$$
\begin{aligned}
& \ddot{\sigma}+\left(\Theta+\frac{\dot{P}_{, X}}{P_{, X}}\right) \dot{\sigma}-\frac{P_{, \sigma}}{P_{, X}} \approx 0, \\
& \dot{\theta}-\frac{P_{, s}}{P_{, X} \dot{\sigma}} \approx 0 .
\end{aligned}
$$

Although they look very similar to the homogeneous equations (115) and (116), these equations are fully inhomogeneous and encode the evolution of nonlinearities on large scales. This limit illustrates the separate universe picture $[36,37]$ where the inhomogeneous universe can be described, on large scales, as juxtaposed Friedmann homogeneous universes.

If, so far, the order in spatial gradients seems to coincide with the perturbative classification of the previous subsection, it differs however for the term

$$
\nabla^{c} \sigma_{c}^{\perp}=D^{c} \sigma_{c}^{\perp}+a^{c} \sigma_{c}^{\perp},
$$

which is first order perturbatively but second order in spatial gradients, at least for the first term on the right hand side, since $\sigma_{c}^{\perp}$ is already first order in spatial gradients. For the second term, it has been shown in [21] that $u^{a}$ can be chosen so that $a^{c}$ is at least first order in spatial gradients. We will explicitly verify it in the next section by working in a coordinate system.

With these prescriptions, the evolution equation of $\sigma_{a}^{\perp}$ and $s_{a}$ obtained at lowest order in spatial gradients become

$$
\begin{aligned}
& \left(\ddot{\sigma}_{a}\right)^{\perp}+\left[\Theta+\frac{c_{s}^{2}}{P_{, X}}\left(\frac{P_{, X}}{c_{s}^{2}}\right)^{\cdot}\right]\left(\dot{\sigma}_{a}\right)^{\perp}+\frac{c_{s}^{2}}{P_{, X}}\left[-P_{, \sigma \sigma}-P_{, X} \dot{\theta}^{2}+e^{-3 \alpha}\left(e^{3 \alpha} \dot{\sigma} P_{, X \sigma}\right)^{\cdot}\right] \sigma_{a}^{\perp}+\dot{\sigma} c_{s}^{2} D_{a} \Theta \\
& \approx \quad \Xi \dot{s}_{a}+\left[\dot{\Xi}-\Xi\left(2 \frac{\dot{c}_{s}}{c_{s}}-\frac{P_{, \sigma}}{P_{, X} \dot{\sigma}}\right)\right] s_{a}
\end{aligned}
$$


and

$$
\ddot{s}_{a}+\left(\Theta+\frac{\dot{P}_{, X}}{P_{, X}}\right) \dot{s}_{a}+\left(\mu_{s}^{2}+\frac{\Xi^{2}}{c_{s}^{2}}\right) s_{a} \approx-\frac{\Xi}{P_{, X} \dot{\sigma}} \epsilon_{a}
$$

where we have dropped the terms containing $\Pi$ in (100), which are two orders higher than $s_{a}$ in spatial gradients.

We now expand the evolution equation for $\zeta_{a}$, Eq. (109), by neglecting higher-order spatial gradients in the two terms on the right hand side of this equation. The nonadiabatic pressure perturbation becomes

$$
\Gamma_{a} \approx-\epsilon_{a} \frac{\dot{\sigma}}{\dot{\rho}}\left[\left(1+c_{s}^{2}\right) P_{, \sigma}-c_{s}^{2} \dot{\sigma}^{2} P_{, X \sigma}\right]+P_{, X} \dot{\sigma} \Xi s_{a},
$$

while the dissipative nonadiabatic pressure perturbation $\Sigma_{a}$ can be completely dropped, since the dissipative term $\mathcal{D}$ is at least second order in the spatial gradients and thus $\Sigma_{a}$ is third order in the spatial gradients. Equation (109) therefore becomes, on large scales,

$$
\dot{\zeta}_{a} \approx-\frac{1}{3 P_{, X}^{2} \dot{\sigma}^{3}}\left[\left(1+c_{s}^{2}\right) P_{, \sigma}-c_{s}^{2} \dot{\sigma}^{2} P_{, X \sigma}\right] \epsilon_{a}-\frac{\Theta \Xi}{3 \dot{\sigma}} s_{a} .
$$

Note that the lowest order limit in spatial gradients of the evolution equations of $\sigma_{a}^{\perp}, s_{a}$ and $\zeta_{a}$, respectively Eqs. (125-126) and (128), are similar to their linear counterparts except for the terms $D_{a}\left(D^{c} \sigma_{c}^{\perp}\right)$ and $D_{a}\left(D^{c} s_{c}\right)$, which are third order in spatial gradients and therefore negligible in the spatial gradient expansion. This is because in these equations the terms that are higher than linear order in the perturbative expansion turn out to be also higher than first order in the spatial gradient expansion.

We now concentrate our attention on the comoving energy density perturbation, $\epsilon_{a}$, defined in Eq. (91). Until now we have only made use of the Klein-Gordon equations of the scalar fields. However, in order to study the behavior of the comoving energy density, we will now make use of the Einstein equations, in particular of the so-called constraint equations. The projection of the Einstein equations along $u^{a}$ yields the energy constraint in a covariant form,

$$
u^{a} G_{a b} u^{b}=8 \pi G \rho .
$$

Assuming that the vector field $u^{a}$ is hypersurface orthogonal, it is possible to use the Gauss-Codazzi equations and the decomposition

$$
D_{b} u_{a}=\sigma_{a b}+\frac{1}{3} \Theta h_{a b},
$$

which is the spatially projected version of (6) (with $\omega_{a b}=0$ since $u^{a}$ is here hypersurface orthogonal), in order to rewrite the energy constraint as

$$
\frac{1}{2}\left({ }^{(3)} R+\frac{2}{3} \Theta^{2}-\sigma_{a b} \sigma^{a b}\right)=8 \pi G \rho,
$$

where ${ }^{(3)} R$ is the intrinsic Ricci scalar of the space-like hypersurfaces orthogonal to $u^{a}$.

The mixed projection of Einstein's equations yields the covariant momentum constraint

$$
u^{b} G_{b c} h_{a}^{c}=8 \pi G q_{a},
$$

which can be rewritten, via Gauss-Codazzi relations and Eq. (130), as

$$
D_{b} \sigma_{a}^{b}-\frac{1}{3} D_{a} \Theta=8 \pi G q_{a} .
$$

By combining the energy and momentum constraints, one obtains the nonlinear covariant version of the generalized Poisson equation, which in the linear theory relates the comoving energy density to the Bardeen's potential defined from the curvature perturbation. Here one finds

$$
\frac{1}{2} D_{a}\left({ }^{(3)} R-\sigma_{b c} \sigma^{b c}\right)+\Theta D_{b} \sigma_{a}^{b}=8 \pi G \tilde{\epsilon}_{a},
$$

where we have introduced on the right hand side the quantity

$$
\tilde{\epsilon}_{a} \equiv D_{a} \rho-\Theta q_{a}=D_{a} \rho+P_{, X} \Theta \dot{\sigma} \sigma_{a}^{\perp} .
$$


This quantity can be seen as an alternative generalization of the comoving energy density perturbation, since, in the linear limit, it is equivalent to $\epsilon_{a}$ defined in (91). In the fully nonlinear case, the two quantities in principle are different: using (107) one gets

$$
\tilde{\epsilon}_{a}-\epsilon_{a}=\frac{1}{\dot{\sigma}}\left(\mathcal{D}-\frac{1}{3} P_{, X} \Theta \Pi\right) \sigma_{a}^{\perp} .
$$

This difference becomes however negligible on large scales,

$$
\epsilon_{a} \approx \tilde{\epsilon}_{a} .
$$

Now, the left hand side of Eq. (134) contains the projected gradient of the Ricci scalar, $D_{a}{ }^{(3)} R$. From its definition in terms of derivatives of the metric, it can be shown that, for a perturbed FLRW universe, this term is of third order in the spatial gradients. Equation (134) can thus be used to show that $\epsilon_{a}$ in Eqs. (106), (126) and (128) can be neglected on large scales, if the shear can also be neglected in this limit. Indeed, on large scales, the shear rapidly decreases in an expanding perturbed FLRW universe. Thus, in this limit the comoving and uniform density integrated expansion perturbations $\zeta_{a}$ and $\mathcal{R}_{a}$ coincide (up to a sign),

$$
\zeta_{a}+\mathcal{R}_{a} \approx 0 .
$$

Furthermore, one can rewrite Eqs. (126) and (128) as a closed coupled system of equations, describing the large-scale nonlinear evolution of adiabatic and entropy perturbations,

$$
\ddot{s}_{a}+\left(\Theta+\frac{\dot{P}_{, X}}{P_{, X}}\right) \dot{s}_{a}+\left(\mu_{s}^{2}+\frac{\Xi^{2}}{c_{s}^{2}}\right) s_{a} \approx 0
$$

and

$$
\dot{\zeta}_{a} \approx-\frac{\Theta \Xi}{3 \dot{\sigma}} s_{a} .
$$

Remarkably, these equations for the covectors $\zeta_{a}$ and $s_{a}$ look very similar to their linear counterparts, studied in [11], although they extend them to describe the full nonlinear evolution for the perturbations. Despite their compact appearence, they exhibit a quite involved dependence on the form of the Lagrangian. For instance, the mass term $\mu_{s}^{2}$, which reduces to $V_{s s}-\dot{\theta}^{2}$ for standard Lagrangian, also depends in our case on the entropic projection of the mixed derivative $P_{, X I}$, a characteristic shared by the important parameter $\Xi$.

As explained in Section 2, we stress again that the equations derived in this subsection in the large scale limit are also valid for two-field DBI inflation with the adequate Lagrangian Eq. (12). In the next sections, always following [21], we will show how to translate our results, obtained within the nonlinear formalism, in the more familiar coordinate-based approach, obtaining quite straightforwardly the evolution equations for the perturbations up to second order.

\section{Linear perturbations}

In this and next section, we relate the covariant approach to the more familiar coordinate based formalism. We analyze the linear perturbations in the present section, and we then consider an expansion up to second order in the next one.

Let us thus introduce generic coordinates $x^{\mu}=\left\{t, x^{i}\right\}$ to describe an almost-FLRW spacetime. Here a prime will denote a partial derivative with respect to the cosmic time $t$, i.e. ${ }^{\prime} \equiv \partial / \partial t$, since the dot has been used till now to denote the Lie derivative with respect to $u^{a}$.

The background spacetime is a FLRW spacetime, endowed with the metric

$$
d s^{2}=\bar{g}_{\mu \nu} d x^{\mu} d x^{\nu}=-d t^{2}+a(t)^{2} \gamma_{i j} d x^{i} d x^{j} .
$$

At linear order, the spacetime geometry is described by the perturbed metric

$$
d s^{2}=\left(\bar{g}_{\mu \nu}+\delta g_{\mu \nu}\right) d x^{\mu} d x^{\nu},
$$

where the components of the metric perturbations can be written as

$$
\delta g_{00}=-2 A, \quad \delta g_{0 i}=a B_{i}, \quad \delta g_{i j}=a^{2} H_{i j} .
$$


We decompose, as usual, $B_{i}$ and $H_{i j}$ in the forms

$$
\begin{aligned}
B_{i} & =\vec{\nabla}_{i} B+B_{i}^{V}, \\
H_{i j} & =-2 \psi \gamma_{i j}+2 \vec{\nabla}_{i} \vec{\nabla}_{j} E+2 \vec{\nabla}_{(i} E_{j)}^{V}+2 E_{i j}^{T},
\end{aligned}
$$

where $B_{i}^{V}$ and $E_{i}^{V}$ are transverse, i.e., $\vec{\nabla}_{i} B^{V i}=0=\vec{\nabla}_{i} E^{V i}$, and $E_{i j}^{T}$ is transverse and traceless, i.e., $\vec{\nabla}_{i} E^{T i j}=0$ and $\gamma^{i j} E_{i j}^{T}=0$. Here $\vec{\nabla}_{i}$ denotes the three-dimensional covariant derivative with respect to the homogeneous spatial metric $\gamma_{i j}$ (which is also used to lower or raise the spatial indices). The matter fields are similarly decomposed into a background and a perturbed part,

$$
\phi^{I}\left(t, x^{i}\right)=\bar{\phi}^{I}(t)+\delta \phi^{I}\left(t, x^{i}\right) .
$$

We now need to specify the components of the unit vector $u^{a}$, which defines the time derivation in our covariant approach. At zeroth order, it is natural to take it orthogonal to the homogeneous slices. At first order, [21] chose, for simplicity, $u^{\mu}$ such that $u_{i}=0$. This implies that, up to first order, the components of $u^{\mu}$ are given by

$$
u^{\mu}=\left\{1-A,-B^{i} / a\right\}
$$

and those of the acceleration vector are given by

$$
a^{\mu}=\left\{0, \vec{\nabla}^{i} A / a^{2}\right\} .
$$

This confirms that $a^{\mu}$ can be considered as first order in spatial gradients, in agreement with the assumption of the previous section.

Since the formalism relies on many covectors, it is useful to first consider a generic covector $Y_{a}$ and work out the components of its time derivative $\dot{Y}_{a}$. To make the explicit calculation, it is convenient to replace in the definition of the Lie derivative (2) the covariant derivatives by partial derivatives and write

$$
\dot{Y}_{a}=u^{b} \partial_{b} Y_{a}+Y_{b} \partial_{a} u^{b}
$$

At zeroth order, the components of $\dot{Y}_{a}$ are simply

$$
\overline{\dot{Y}}_{\mu}=\left\{\bar{Y}_{0}^{\prime}, 0,0,0\right\},
$$

assuming that the spatial components $\bar{Y}_{i}$ vanish so as to respect the symmetries of the geometry. At first order, we get from (149) and (147)

$$
\delta\left(\dot{Y}_{0}\right)=\delta Y_{0}^{\prime}-\left(\bar{Y}_{0} A\right)^{\prime}, \quad \delta\left(\dot{Y}_{i}\right)=\delta Y_{i}^{\prime}-\bar{Y}_{0} \partial_{i} A
$$

Let us now consider, for the special case of two scalar fields, the adiabatic and entropic covectors $\sigma_{a}$ and $s_{a}$, which we have introduced earlier. Note that with the choice of four-velocity $(147) \sigma_{i}^{\perp}=\sigma_{i}$. The background equations of motion can be deduced immediately from Eqs. (63) and (69) and read

$$
\begin{gathered}
\frac{\bar{\sigma}^{\prime \prime}}{\bar{c}_{s}^{2}}+3 H \bar{\sigma}^{\prime}+\frac{\bar{P}_{, X \sigma}}{\bar{P}_{, X}} \bar{\sigma}^{2}-\frac{\bar{P}_{, \sigma}}{\bar{P}_{, X}}=0, \\
\bar{\sigma}^{\prime} \bar{\theta}^{\prime}-\frac{\bar{P}_{, s}}{\bar{P}_{, X}}=0,
\end{gathered}
$$

with

$$
\bar{\sigma}_{0}=\bar{\sigma}^{\prime} \equiv \sqrt{\bar{G}_{I J} \bar{\phi}^{I^{\prime}} \bar{\phi}^{J^{\prime}}}
$$

Note that we used the symbol $\bar{\theta}^{\prime}$ to represent the background value of $\dot{\theta}$. Again, as there is no angle $\theta$, this is merely a notational convenience.

From its definition, Eq. (50), one finds that the spatial components of $\sigma_{a}$ at linear order can be expressed as

$$
\delta \sigma_{i}=\bar{e}_{\sigma I} \partial_{i} \delta \phi^{I}=\partial_{i} \delta \sigma
$$


We use the notation

$$
\delta \sigma \equiv \bar{e}_{\sigma I} \partial_{i} \delta \phi^{I}
$$

in agreement with previous studies in the literature. By using Eq. (151) with (154-155) one finds that the spatial components of the first and second time derivatives are given by

$$
\delta\left(\dot{\sigma}_{i}\right)=\partial_{i}\left(\delta \sigma^{\prime}-\bar{\sigma}^{\prime} A\right), \quad \delta\left(\ddot{\sigma}_{i}\right)=\partial_{i}\left(\delta \sigma^{\prime \prime}-\bar{\sigma}^{\prime} A^{\prime}-2 \bar{\sigma}^{\prime \prime} A\right) .
$$

The same procedure for $s_{a}$ gives

$$
\delta s_{i}=\partial_{i} \delta s, \quad \delta s \equiv \bar{e}_{s I} \partial_{i} \delta \phi^{I},
$$

which also coincides with the notation of [11]. Since $s_{a}$, in contrast to $\sigma_{a}$, has no longitudinal component, $\bar{s}_{0}=0$ and the spatial components of $\dot{s}_{a}$ and $\ddot{s}_{a}$ are simply

$$
\delta\left(\dot{s}_{i}\right)=\partial_{i} \delta s^{\prime}, \quad \delta\left(\ddot{s}_{i}\right)=\partial_{i} \delta s^{\prime \prime} .
$$

Plugging the explicit components (157) and (159) into the linearized equations for $\sigma_{a}$ and $s_{a}$, given by (117) and (118) respectively, one easily obtains the linearized equations for $\delta \sigma$ and $\delta s$. These read, respectively,

$$
\begin{aligned}
\delta \sigma^{\prime \prime}+ & {\left[3 H+\frac{\bar{c}_{s}^{2}}{\bar{P}_{, X}}\left(\frac{\bar{P}_{, X}}{\bar{c}_{s}^{2}}\right)^{\prime}\right] \delta \sigma^{\prime}+\frac{\bar{c}_{s}^{2}}{\bar{P}_{, X}}\left[-\bar{P}_{, \sigma \sigma}-\bar{P}_{, X} \bar{\theta}^{\prime 2}+e^{-3 \bar{\alpha}}\left(e^{3 \bar{\alpha}} \bar{\sigma}^{\prime} \bar{P}_{, X \sigma}\right)^{\prime}\right] \delta \sigma } \\
& -\bar{\sigma}^{\prime}\left[A^{\prime}+\left(2 \frac{\bar{\sigma}^{\prime \prime}}{\bar{\sigma}^{\prime}}+3 H\left(1+\bar{c}_{s}^{2}\right)+\frac{\bar{c}_{s}^{2}}{\bar{P}_{, X}}\left(\frac{\bar{P}_{, X}}{\bar{c}_{s}^{2}}\right)^{\prime}\right) A+3 \bar{c}_{s}^{2} \psi^{\prime}-\bar{c}_{s}^{2} \vec{\nabla}^{2}\left(E^{\prime}-B / a\right)\right]-\frac{\bar{c}_{s}^{2}}{a^{2}} \vec{\nabla}^{2} \delta \sigma \\
= & \Xi \delta s^{\prime}+\left[\bar{\Xi}^{\prime}-\bar{\Xi}\left(2 \frac{\bar{c}_{s}^{\prime}}{\bar{c}_{s}}-\frac{\bar{P}_{, \sigma}}{\bar{P}_{, X} \bar{\sigma}^{\prime}}\right)\right] \delta s
\end{aligned}
$$

and

$$
\delta s^{\prime \prime}+\left(3 H+\frac{\bar{P}_{, X}^{\prime}}{\bar{P}_{, X}}\right) \delta s^{\prime}+\left(\bar{\mu}_{s}^{2}+\frac{\bar{\Xi}^{2}}{\bar{c}_{s}^{2}}\right) \delta s-\frac{1}{a^{2}} \vec{\nabla}^{2} \delta s=-\frac{\bar{\Xi}}{\bar{P}_{, X} \bar{\sigma}^{\prime}} \delta \epsilon,
$$

where we have used

$$
\bar{\Theta}=3 H, \quad \delta \Theta=-3 H A-3 \psi^{\prime}+\vec{\nabla}^{2}\left(E^{\prime}-B / a\right) .
$$

In the latter equation we have introduced the first-order comoving energy density perturbation $\delta \epsilon$, defined by

$$
\delta \epsilon_{i}=\partial_{i} \delta \epsilon, \quad \delta \epsilon \equiv \delta \rho-\frac{\bar{\rho}^{\prime}}{\bar{\sigma}^{\prime}} \delta \sigma
$$

which follows from the definition (91) of $\epsilon_{a}$. Using

$$
\begin{aligned}
\bar{\rho} & =\bar{P}_{, X} \bar{\sigma}^{\prime 2}-\bar{P}, \\
\delta \rho & =\frac{\bar{P}_{, X} \bar{\sigma}^{\prime}}{\bar{c}_{s}^{2}}\left(\delta \sigma^{\prime}-\bar{\sigma}^{\prime} A-\bar{\Xi} \delta s\right)+\left(\bar{P}_{, X \sigma} \bar{\sigma}^{\prime 2}-\bar{P}_{, \sigma}\right) \delta \sigma,
\end{aligned}
$$

one sees that $\delta \epsilon$ can be expressed as

$$
\delta \epsilon=\frac{\bar{P}_{, X} \bar{\sigma}^{\prime}}{\bar{c}_{s}^{2}}\left[\delta \sigma^{\prime}-\bar{\sigma}^{\prime} A-\frac{\bar{\sigma}^{\prime \prime}}{\bar{\sigma}^{\prime}} \delta \sigma-\bar{\Xi} \delta s\right] .
$$

Moreover, linearizing the spatial components of the energy constraint (131) yields

$$
3 H\left(\psi^{\prime}+H A\right)-\frac{1}{a^{2}} \vec{\nabla}^{2}\left[\psi+H\left(a^{2} E^{\prime}-a B\right)\right]=-4 \pi G \delta \rho,
$$

while the momentum constraint (133) gives, since $\delta q_{i}=-\bar{P}_{, X} \bar{\sigma}^{\prime} \partial_{i} \delta \sigma$,

$$
\psi^{\prime}+H A=4 \pi G \bar{P}_{, X} \bar{\sigma}^{\prime} \delta \sigma .
$$


Combining the two above constraints yields the relativistic Poisson-like equation

$$
\frac{1}{a^{2}} \vec{\nabla}^{2}\left[\psi+H\left(a^{2} E^{\prime}-a B\right)\right]=4 \pi G \delta \epsilon,
$$

which can also be directly obtained by linearizing the spatial components of Eq. (134). This equation shows that the comoving energy density perturbation $\delta \epsilon$ is second order in the spatial gradients, and thus negligible on large scales in Eq. (161).

The quantity $\delta \sigma$ is not gauge-invariant, in contrast with $\delta s$. This is why it is useful to consider the gauge invariant Sasaki-Mukhanov variable $Q_{\mathrm{SM}}$, defined as [38]

$$
Q_{\mathrm{SM}} \equiv \delta \sigma+\frac{\bar{\sigma}^{\prime}}{H} \psi
$$

Note that the above traditional definition does not follow exactly from the definition of $Q_{a}$ given earlier in Eq. (105). Indeed, from $Q_{a}$, one can extract a scalar quantity $Q$ defined as

$$
Q_{i}=\partial_{i} Q, \quad Q \equiv \delta \sigma-\frac{\bar{\sigma}^{\prime}}{H} \delta \alpha,
$$

where $\delta \alpha$ can be written in terms of metric perturbations by making use of Eqs. (35) and (162) (see [19]),

$$
\delta \alpha=-\psi+\frac{1}{3} \int \vec{\nabla}^{2}\left(E^{\prime}-B / a\right) d t
$$

Thus the scalar variable $Q$ coincides with $Q_{\mathrm{SM}}$ only in the large-scale limit.

In the flat gauge ${ }^{5}$, defined by $\hat{\psi} \equiv 0, \delta \sigma$ coincides with $Q_{\mathrm{SM}}$,

$$
\hat{\delta \sigma}=Q_{\mathrm{SM}}
$$

In this gauge, it is possible to use the momentum constraint equations (167) to derive the metric perturbation $A$ as a function of $Q_{\mathrm{SM}}$,

$$
\hat{A}=-\frac{H^{\prime}}{H \bar{\sigma}^{\prime}} Q_{\mathrm{SM}}
$$

and one can write the Poisson equation (168) as

$$
\vec{\nabla}^{2}\left(\hat{E}^{\prime}-\hat{B} / a\right)=-\frac{H^{\prime}}{H \bar{c}_{s}^{2} \bar{\sigma}^{\prime}}\left[Q_{\mathrm{SM}}^{\prime}+\left(\frac{H^{\prime}}{H}-\frac{\bar{\sigma}^{\prime \prime}}{\bar{\sigma}^{\prime}}\right) Q_{\mathrm{SM}}-\Xi \delta s\right],
$$

where we have used the expression (165) specialized to the flat gauge. [11],

By replacing Eqs. (173) and (174) into the evolution equation of $\hat{\delta \sigma}$, one finds the evolution equation of $Q_{\mathrm{SM}}$

$$
Q_{\mathrm{SM}}^{\prime \prime}+\left(3 H+\frac{\bar{c}_{s}^{2}}{\bar{P}_{, X}}\left(\frac{\bar{P}_{, X}}{\bar{c}_{s}^{2}}\right)^{\prime}\right) Q_{\mathrm{SM}}^{\prime}+\left(\mu_{\sigma}^{2}-\frac{\bar{c}_{s}^{2} \vec{\nabla}^{2}}{a^{2}}\right) Q_{\mathrm{SM}}=(\Xi \delta s)^{\prime}-\Xi\left(\frac{\left(H \bar{c}_{s}^{2}\right)^{\prime}}{H \bar{c}_{s}^{2}}-\frac{\bar{P}_{, \sigma}}{\bar{\sigma}^{\prime} \bar{P}_{, X}}\right) \delta s,
$$

with

$$
\mu_{\sigma}^{2} \equiv-\left[\frac{\left(\bar{\sigma}^{\prime} / H\right)^{\prime}}{\bar{\sigma}^{\prime} / H}\right]^{\prime}-\left(3 H+\frac{\bar{c}_{s}^{2}}{\bar{P}_{, X}}\left(\frac{\bar{P}_{, X}}{\bar{c}_{s}^{2}}\right)^{\prime}+\frac{\left(\bar{\sigma}^{\prime} / H\right)^{\prime}}{\bar{\sigma}^{\prime} / H}\right) \frac{\left(\bar{\sigma}^{\prime} / H\right)^{\prime}}{\bar{\sigma}^{\prime} / H}
$$

When one considers only large scales, the expression (174) reduces to

$$
Q_{\mathrm{SM}}^{\prime}+\left(\frac{H^{\prime}}{H}-\frac{\bar{\sigma}^{\prime \prime}}{\bar{\sigma}^{\prime}}\right) Q_{\mathrm{SM}}-\bar{\Xi} \delta s \approx 0
$$

which means that there exists a first integral for the quantity $Q_{\mathrm{SM}}$ and that the second-order equation of motion (175) is not necessary in this limit. In fact, one can easily check that the large-scale limit of (175) is an automatic consequence of the first integral (177).

\footnotetext{
${ }^{5}$ In the following, a hat indicates that the corresponding quantity is evaluated in the flat gauge.
} 
Let us now consider the evolution equation for $\zeta_{a}$ with two scalar fields. The spatial components of $\zeta_{a}$, at linear order, are given by [19]

$$
\delta \zeta_{i}=\partial_{i} \zeta, \quad \zeta \equiv \delta \alpha-\frac{H}{\bar{\rho}^{\prime}} \delta \rho
$$

From Eq. (171) the scalar variable $\zeta$ is thus related to the Bardeen gauge invariant variable $\zeta_{\mathrm{B}}$, defined as $[39,40]$

$$
\zeta_{\mathrm{B}} \equiv-\psi-\frac{H}{\bar{\rho}^{\prime}} \delta \rho
$$

in a similar way to how $Q$ is related to $Q_{\mathrm{SM}}$, and on large scales these two quantities coincide.

According to Eq. (151), the spatial components of $\dot{\zeta}_{a}$ are

$$
\delta\left(\dot{\zeta}_{i}\right)=\partial_{i} \zeta^{\prime}
$$

On large scales, one then finds the relation

$$
\zeta^{\prime} \approx-\frac{H}{\bar{\sigma}^{\prime}} \Xi \delta s
$$

Note also that from Eq. (106) there is a simple relation between $\zeta$ and $\mathcal{R}$,

$$
\zeta+\mathcal{R}=-\frac{\bar{\alpha}^{\prime}}{\bar{\rho}^{\prime}} \delta \epsilon
$$

which shows that $\zeta$ and $-\mathcal{R}$ coincide on large scales.

In conclusion, all the equations in this section, derived directly from the covariant formalism, exactly reproduce the linear results of [11]. In particular, according to Eqs. (161) and (175), whereas entropic perturbations are sensitive to the usual Hubble horizon $(k \approx a H)$, adiabatic perturbations are amplified at sound horizon crossing, $c_{s} k \approx a H$. As shown in [12, 13], these equations are not directly applicable to two-field DBI inflation, whose Lagrangian is not of the form studied in this paper. Despite that, in that situation, it turns out that Eq. (175) for the adiabatic perturbation is still valid and that Eq. (161) for the entropy perturbation is minimally modified, simply replacing the gradient term $\frac{1}{a^{2}} \vec{\nabla}^{2} \delta s$ by $\frac{\bar{c}_{s}^{2}}{a^{2}} \vec{\nabla}^{2} \delta s$, for the adequate Lagrangian Eq. (12), with the consequence that both types of perturbations are amplified at sound horizon crossing.

In the next section we turn to the second-order perturbations, and show the power of the method we are using to derive new results.

\section{$7 \quad$ Second order perturbations}

In this section we consider second-order perturbations (for a recent review, see [41]). We will thus decompose any scalar quantity $Y$ as

$$
Y\left(t, x^{i}\right) \equiv \bar{Y}(t)+\delta Y^{(1)}\left(t, x^{i}\right)+\delta Y^{(2)}\left(t, x^{i}\right)
$$

where $\bar{Y}(t)$ is the background part and $\delta Y^{(1)}$ and $\delta Y^{(2)}$ are respectively the first and second-order contributions (note that we do not follow here the convention of including a numerical factor $1 / 2$ in front of the second-order contribution). In our subsequent equations, to simplify the notation, we will often omit the index (1) for the first-order quantities, unless it is required for clarity reasons.

Our main purpose will be to expand our equations governing $\sigma_{a}, s_{a}$ and $\zeta_{a}$ at second order in the perturbations. We start with a brief discussion on the gauge invariance of the curvature perturbation. As shown in [19], the second-order expression of the spatial components $\zeta_{i}$ can be written, after some manipulations, in the form

$$
\zeta_{i}^{(2)}=\partial_{i} \zeta^{(2)}+\frac{\delta \rho}{\bar{\rho}^{\prime}} \partial_{i} \zeta^{(1) \prime}
$$

with

$$
\zeta^{(2)} \equiv \delta \alpha^{(2)}-\frac{H}{\bar{\rho}^{\prime}} \delta \rho^{(2)}-\frac{\delta \rho}{\bar{\rho}^{\prime}}\left[\zeta^{(1) \prime}+\frac{1}{2}\left(\frac{H}{\bar{\rho}^{\prime}}\right)^{\prime} \delta \rho\right],
$$

and $\zeta^{(1)}$ being given in Eq. (178). In [21] it has been shown that $\zeta^{(2)}$ is gauge invariant on large scales: their proof holds identical in our case and we then refer the reader to this paper for more details. We now pass to discuss the evolution equations for adiabatic and entropy fields up to second order, and analyze how they source the curvature perturbation on large scales. The reader should keep in mind that the following discussion, restricted to large scales, is applicable to two-field DBI models on using the adequate Lagrangian Eq. (12). 


\subsection{Adiabatic and entropy fields}

Here we derive the evolution equations for the adiabatic and entropy field perturbations at second order. We will restrict ourselves to large scales, a situation in which the equations become more tractable, starting from the equations expanded in spatial gradients discussed in Sec. 5.2. For convenience, we have collected in the appendix various background and first-order expressions that will be used in the rest of this section.

Second-order evolution for the perturbations when several scalars are involved has been first studied in detail by Malik in [42] in the large-scale limit and with canonical kinetic terms (see also [43, 44, 45]), while it has been analyzed in $[46,47]$ using the separate universe approach. The second-order decomposition into adiabatic and entropy components however, has only been derived in [21], in the case of standard kinetic terms (see [48] for a recent application and $[49,50]$ for related studies). This decomposition can easily be derived since the fully nonlinear adiabatic and entropy components have already been identified. We will show how to extend this decomposition into adiabatic and entropy components to the case of non-trivial metric in field space, and use it to derive the corresponding evolution equations for these modes.

In order to pursue the decomposition, one starts by expanding the definition of $\sigma_{a}$ in Eq. (50) at second order. After some straightforward manipulations, one can write the spatial components of $\sigma_{i}$ as

$$
\delta \sigma_{i}^{(2)}=\partial_{i}\left[\bar{e}_{\sigma I} \delta^{(2)} \phi^{I}+\frac{1}{2} \bar{e}_{\sigma}^{J} \bar{\Gamma}_{J I K}\left(\bar{e}_{\sigma}^{K} \bar{e}_{\sigma}^{I} \delta \sigma^{2}+\bar{e}_{s}^{K} \bar{e}_{s}^{I} \delta s^{2}+2 \bar{e}_{\sigma}^{K} \bar{e}_{s}^{I} \delta \sigma \delta s\right)\right]+\frac{1}{\bar{\sigma}^{\prime}}\left(\delta s^{\prime}+\bar{\theta}^{\prime} \delta \sigma\right) \partial_{i} \delta s
$$

To deal with the term $\delta s^{\prime} \partial_{i} \delta s$, which cannot be written as a total gradient, it is convenient to introduce the spatial vector

$$
V_{i} \equiv \frac{1}{2}\left(\delta s \partial_{i} \delta s^{\prime}-\delta s^{\prime} \partial_{i} \delta s\right)=-\delta s^{\prime} \partial_{i} \delta s+\frac{1}{2} \partial_{i}\left(\delta s \delta s^{\prime}\right)
$$

which vanishes when $\delta s^{\prime}$ and $\delta s$ have the same spatial dependence, i.e., $\delta s^{\prime}=f(t) \delta s$.

By expanding also the definition of $s_{a}$ in Eq. (51), one finds, for $s_{i}$ and $\sigma_{i}$, respectively,

$$
\begin{aligned}
\delta \sigma_{i}^{(2)} & =\partial_{i} \delta \sigma^{(2)}+\frac{\bar{\theta}^{\prime}}{\bar{\sigma}^{\prime}} \delta \sigma \partial_{i} \delta s-\frac{1}{\bar{\sigma}^{\prime}} V_{i}, \\
\delta s_{i}^{(2)} & =\partial_{i} \delta s^{(2)}+\frac{\delta \sigma}{\bar{\sigma}^{\prime}} \partial_{i} \delta s^{\prime},
\end{aligned}
$$

with

$$
\begin{aligned}
\delta \sigma^{(2)} & \equiv \bar{e}_{\sigma I} \delta^{(2)} \phi^{I}+\frac{1}{2} \bar{e}_{\sigma}^{J} \bar{\Gamma}_{J I K}\left(\bar{e}_{\sigma}^{K} \bar{e}_{\sigma}^{I} \delta \sigma^{2}+\bar{e}_{s}^{K} \bar{e}_{s}^{I} \delta s^{2}+2 \bar{e}_{\sigma}^{K} \bar{e}_{s}^{I} \delta \sigma \delta s\right)+\frac{1}{2 \bar{\sigma}^{\prime}} \delta s \delta s^{\prime} \\
\delta s^{(2)} & \equiv \bar{e}_{s I} \delta^{(2)} \phi^{I}+\frac{1}{2} \bar{e}_{s}^{J} \bar{\Gamma}_{J I K}\left(\bar{e}_{\sigma}^{K} \bar{e}_{\sigma}^{I} \delta \sigma^{2}+\bar{e}_{s}^{K} \bar{e}_{s}^{I} \delta s^{2}+2 \bar{e}_{\sigma}^{K} \bar{e}_{s}^{I} \delta \sigma \delta s\right)-\frac{\delta \sigma}{\bar{\sigma}^{\prime}}\left(\delta s^{\prime}+\frac{\bar{\theta}^{\prime}}{2} \delta \sigma\right)
\end{aligned}
$$

The form of the right hand side of Eq. (189) has been chosen by analogy with the form (184). Since $s_{a}$ vanishes at zeroth order, arguments similar to those used in [21] to prove gauge invariance for $\zeta^{(2)}$ ensure that $\delta s^{(2)}$, defined in Eq. (191), is also gauge invariant on large scales. The form of $\delta s^{(2)}$ is, then, forced by our covariant definition. Let us remark that the non-flat nature of the field space metric manifests itsef in the appearance of the terms with Christoffel symbols in $\delta \sigma^{(2)}$ and $\delta s^{(2)}$. Otherwise, these are formally identical to the corresponding definitions in [21].

Note that $\delta s_{i}^{(2)}$ contains the first-order adiabatic perturbation. This is due to the fact that the adiabatic and entropy components are defined locally: whereas the first-order components are defined with respect to a background basis in field space, which is only time dependent, the second-order components will be sensitive to the first-order fluctuations of the field space basis, which can be expressed in terms of the first-order adiabatic and entropy components. Then, the adiabatic component $\sigma_{a}$ does not vanish at zeroth order and $\delta \sigma^{(2)}$ is not a gauge invariant variable. It is then useful to consider our generalization of the Sasaki-Mukhanov variable, $Q_{a}$, defined in Eq. (105). Its spatial components can be expanded at second order in the perturbations, similarly to what we have done with $\zeta_{a}$,

$$
Q_{i}^{(2)}=\partial_{i} Q^{(2)}+\frac{\delta \alpha}{H} \partial_{i} Q^{\prime}+\frac{\bar{\theta}^{\prime}}{\bar{\sigma}^{\prime}} Q \partial_{i} \delta s-\frac{1}{\bar{\sigma}^{\prime}} V_{i}
$$


where $Q^{(2)}$ is defined as

$$
Q^{(2)} \equiv \delta \sigma^{(2)}-\frac{\bar{\sigma}^{\prime}}{H} \delta \alpha^{(2)}-\frac{\delta \alpha}{H}\left[Q^{(1) \prime}+\frac{1}{2}\left(\frac{\bar{\sigma}^{\prime}}{H}\right)^{\prime} \delta \alpha-\bar{\theta}^{\prime} \delta s\right] .
$$

From this expression it is natural to define

$$
Q_{\mathrm{SM}}^{(2)} \equiv \delta \sigma^{(2)}+\frac{\bar{\sigma}^{\prime}}{H}\left(\psi^{(2)}+\psi^{2}\right)+\frac{\psi}{H}\left[Q_{\mathrm{SM}}^{(1)}{ }^{\prime}-\frac{1}{2}\left(\frac{\bar{\sigma}^{\prime}}{H}\right)^{\prime} \psi-\bar{\theta}^{\prime} \delta s\right]
$$

as the local part of the scalar gauge invariant second-order Sasaki-Mukhanov variable. Restricted to a single scalar field, this definition coincides with the one given in [42]. Note that we cannot write Eq. (192) in the same form as Eq. (184) because the last two terms on the right hand side cannot be written as a total spatial gradient.

The second-order (in time) evolution of $\sigma_{a}$ is given by Eq. (125). However, on large scales we do not need to compute a second-order differential equation because the adiabatic evolution is governed by a first integral, as in the linear case. This first integral is obtained directly from the constraint equations and it is not necessary to expand (125) at second order in the perturbations.

In order to compute this first integral we need the second-order energy and momentum constraints, which can be derived by expanding Eqs. (131) and (133) and by using

$$
\delta \Theta^{(2)} \approx \frac{9}{2} H A^{2}+3 A \psi^{\prime}-6 \psi \psi^{\prime}-3 H A^{(2)}-3 \psi^{(2)^{\prime}} .
$$

On large scales, one can write the second-order energy constraint equation as [21]

$$
3 H\left[H A^{(2)}+\psi^{(2) \prime}+2 \psi \psi^{\prime}-\frac{1}{2} \frac{\psi^{\prime 2}}{H}-2 A\left(H A+\psi^{\prime}\right)\right] \approx-4 \pi G \delta \rho^{(2)},
$$

where $\delta \rho^{(2)}$ can be calculated using (note that the non-flat nature of the field space metric simply manifests itsef in the presence of the Riemann tensor)

$$
\begin{aligned}
\delta^{(2)} \dot{\sigma}^{2} & =\bar{\sigma}^{\prime 2}\left(4 A^{2}-2 A^{(2)}\right)-4 A \bar{\sigma}^{\prime}\left(\delta \sigma^{\prime}-\bar{\theta}^{\prime} \delta s\right)+\left(\delta \sigma^{\prime}-\bar{\theta}^{\prime} \delta s\right)^{2}+\left(\delta s^{\prime}+\bar{\theta}^{\prime} \delta \sigma\right)^{2} \\
& -2 \bar{\sigma}^{\prime} \bar{\theta}^{\prime} \delta s^{(2)}+2 \bar{\sigma}^{\prime} \delta \sigma^{(2) \prime}-2 \bar{\theta}^{\prime} \delta \sigma\left(\delta s^{\prime}+\frac{\bar{\theta}^{\prime}}{2} \delta \sigma\right)-\bar{\sigma}^{\prime}\left(\frac{1}{\bar{\sigma}^{\prime}} \delta s \delta s^{\prime}\right)^{\prime} \\
& +\bar{\sigma}^{\prime 2} \bar{e}_{\sigma}^{I} \bar{e}_{\sigma}^{J} \bar{e}_{s}^{K} \bar{e}_{s}^{L} \bar{R}_{K I J L} \delta s^{2} .
\end{aligned}
$$

Explicitely, this gives

$$
\delta \rho^{(2)} \approx \frac{\bar{P}_{, X}}{\bar{c}_{s}^{2}}\left(\bar{\sigma}^{\prime} \delta \sigma^{(2) \prime}-{\bar{\sigma}^{\prime}}^{2} A^{(2)}-\bar{\sigma}^{\prime} \bar{\Xi} \delta s^{(2)}\right)+\left({\bar{\sigma}^{\prime}}^{2} \bar{P}_{, X \sigma}-\bar{P}_{, \sigma}\right) \delta \sigma^{(2)}+\Lambda_{\rho}
$$

where $\Lambda_{\rho}$ is a quadratic function of $A, \delta \sigma^{(1)}, \delta s^{(1)}$, and their first derivatives,

$$
\begin{aligned}
& \Lambda_{\rho} \equiv \frac{\bar{P}_{, X}}{2 \bar{c}_{s}^{2}} \delta \sigma^{\prime}\left(\delta \sigma^{\prime}-2 \bar{\theta}^{\prime} \delta s\right)+\frac{\delta s^{\prime}}{\bar{\sigma}^{\prime}}\left[\frac{1}{2 \bar{c}_{s}^{2}}\left(\left(1+\bar{c}_{s}^{2}\right) \bar{P}_{, \sigma}-\bar{c}_{s}^{2} \bar{\sigma}^{\prime 2} \bar{P}_{, X \sigma}\right) \delta s-\left(\bar{P}_{, s}-\bar{\sigma}^{\prime 2} \bar{P}_{, X s}\right) \delta \sigma\right] \\
& -\frac{1}{2}\left[\bar{P}_{, \sigma \sigma}-\bar{\sigma}^{\prime 2} \bar{P}_{, X \sigma \sigma}+\frac{\bar{\theta}^{\prime}}{\bar{\sigma}^{\prime}}\left(\bar{P}_{, s}-\bar{\sigma}^{\prime 2} \bar{P}_{, X s}\right)\right] \delta \sigma^{2}+\left({\bar{\sigma}^{\prime}}^{2} \bar{P}_{, X \sigma s}-\bar{P}_{, \sigma s}\right) \delta s \delta \sigma \\
& +\frac{1}{2}\left[\bar{\sigma}^{\prime 2} \bar{P}_{, X s s}-\bar{P}_{, s s}+\frac{\bar{P}_{, X}}{\bar{c}_{s}^{2}}\left(\bar{\mu}_{s}^{2}+\frac{\bar{\Xi}^{2}}{\bar{c}_{s}^{2}}+{\bar{\theta}^{\prime}}^{2}+\bar{\sigma}^{\prime 2} \bar{e}_{\sigma}^{I} \bar{e}_{\sigma}^{J} \bar{e}_{s}^{K} \bar{e}_{s}^{L} \bar{R}_{K I J L}\right)\right] \delta s^{2} \\
& +\frac{1}{2}\left(\bar{P}_{, X X X} \bar{\sigma}^{\prime 4}+3 \bar{P}_{, X X}{\bar{\sigma}^{\prime}}^{2}\right)\left(\delta \sigma^{\prime}-\bar{\theta}^{\prime} \delta s-A \bar{\sigma}^{\prime}\right)^{2} \\
& +\bar{\sigma}^{\prime}\left[\left(\bar{P}_{, X X \sigma}{\bar{\sigma}^{\prime}}^{2}+\bar{P}_{, X \sigma}\right) \delta \sigma+\left(\bar{P}_{, X X s}{\bar{\sigma}^{\prime}}^{2}+\bar{P}_{, X s}\right) \delta s-\frac{2 \bar{P}_{, X}}{\bar{c}_{s}^{2}} A\right]\left(\delta \sigma^{\prime}-\bar{\theta}^{\prime} \delta s-A \bar{\sigma}^{\prime}\right) .
\end{aligned}
$$


The second-order momentum constraint equation reads [21]

$$
\partial_{i}\left[H A^{(2)}+\psi^{(2) \prime}+2 \psi \psi^{\prime}-\frac{1}{2} H A^{2}-A\left(H A+\psi^{\prime}\right)\right] \approx-4 \pi G \delta q_{i}^{(2)}
$$

where the second-order momentum $\delta q_{i}^{(2)}$ is given, from Eq. (94), by

$$
\delta q_{i}^{(2)}=-\partial_{i}\left[\bar{P}_{, X} \bar{\sigma}^{\prime} \delta \sigma^{(2)}+\frac{\bar{P}_{, X \sigma} \bar{\sigma}^{\prime}}{2} \delta \sigma^{2}+\frac{\bar{P}_{, X}}{2 \bar{c}_{s}^{2}} \frac{\bar{\sigma}^{\prime \prime}}{\bar{\sigma}^{\prime}} \delta \sigma^{2}+\bar{P}_{, X} \bar{\theta}^{\prime} \delta \sigma \delta s\right]-\frac{1}{\bar{\sigma}^{\prime}} \delta \epsilon \partial_{i} \delta \sigma+\bar{P}_{, X} V_{i} .
$$

As already noticed in [31] for large scales, $\delta q_{i}^{(2)}$ cannot be written as a total gradient when several scalar fields are present. After neglecting $\delta \epsilon$ on large scales in the above equation, this is manifest because of the presence of $V_{i}$. This implies that, in principle, if $V_{i}$ does not vanish, one cannot define at second-order a comoving gauge, i.e., such that $\delta q_{i}^{(1)}=0$ and $\delta q_{i}^{(2)}=0$, in contrast with the linear theory or the single-field case.

However, it is instructive to derive the evolution equation for $V_{i}$ on large scales by using the linear evolution equation for $\delta s$, Eq. (161), neglecting the gradient term and $\delta \epsilon$ at first order. One finds a simple expression that generalizes the corresponding result of [21] to the case of non-canonical kinetic terms

$$
V_{i}^{\prime}+\left(3 H+\frac{\bar{P}_{, X}^{\prime}}{\bar{P}_{, X}}\right) V_{i}=0
$$

This implies that, in an expanding universe, $\bar{P}_{, X} V_{i}$ will decay like $a^{-3}$ and rapidly become negligible even if it is nonzero initially. Consequently, in an expanding universe one can in practice ignore $V_{i}$ on large scales and thus define, in an approximate sense, a comoving gauge at second order, which coincides with $\delta \sigma^{(1)}=0=\delta \sigma^{(2)}$. In this approximate comoving gauge, the momentum (201) can be written as a total gradient. In the rest of the paper, in order to remain as general as possible, we will keep the term $V_{i}$.

Similarly to the first-order case, it is possible to combine the energy and momentum constraint equations and derive the relativistic Poisson-like equation analogous to Eq. (168), which corresponds to the expansion, at second order and on large scales, of Eq. (134). By expanding Eq. (135) and using (137), one has

$$
\delta \epsilon_{i}^{(2)} \approx \partial_{i} \delta \rho^{(2)}-3 H \delta q_{i}^{(2)}-\delta \Theta \delta q_{i} \approx 0,
$$

where the last approximate equality is a consequence of Eqs. (162), (167), (196) and (200) and confirms our conclusion of Sec. 5.2 in a covariant context, namely that we can neglect $\epsilon_{a}$ on large scales. The second-order spatial components of $\epsilon_{a}$ defined in Eq. (91), can be decomposed as

$$
\delta \epsilon_{i}^{(2)}=\partial_{i} \delta \epsilon^{(2)}+\frac{\delta \sigma}{\bar{\sigma}^{\prime}} \partial_{i} \delta \epsilon^{(1) \prime}-3 H \bar{P}_{, X} V_{i}
$$

with $\delta \epsilon^{(2)}$ defined by

$$
\delta \epsilon^{(2)} \equiv \delta \rho^{(2)}-\frac{\bar{\rho}^{\prime}}{\bar{\sigma}^{\prime}} \delta \sigma^{(2)}-\frac{\delta \sigma}{\bar{\sigma}^{\prime}}\left[\delta \epsilon^{(1) \prime}+\frac{1}{2}\left(\frac{\bar{\rho}^{\prime}}{\bar{\sigma}^{\prime}}\right)^{\prime} \delta \sigma+\frac{\bar{\rho}^{\prime}}{\bar{\sigma}^{\prime}} \bar{\theta}^{\prime} \delta s\right] .
$$

It is only when $V_{i}$ is negligible that the quantity $\delta \epsilon^{(2)}$ can be interpreted as the comoving energy density at second order. Otherwise, as discussed before, the comoving gauge cannot be defined.

Using the decomposition (204) and the fact that $\delta \epsilon^{(1)}$ is negligible on large scales, Eq. (203) can be written as

$$
\partial^{2} \delta \epsilon^{(2)} \approx 3 H \bar{P}_{, X} \partial^{i} V_{i}
$$

When $V_{i}$ is negligible, and only then, one finds that, like at first order, the second-order comoving energy density is negligible on large scales,

$$
\delta \epsilon^{(2)} \approx 0 .
$$

Having discussed the general properties of the second-order constraint equations, we can now derive the evolution equation of the gauge-invariant adiabatic component $Q_{\mathrm{SM}}^{(2)}$. Similarly to what we have shown in the 
previous section for the first-order variables, the simplest way to derive an equation satisfied by $Q_{\mathrm{SM}}^{(2)}$ is to work in the flat gauge $\hat{\psi}^{(1)}=0=\hat{\psi}^{(2)}$, where $\delta \sigma^{(2)}$ reduces to $Q_{\mathrm{SM}}^{(2)}$,

$$
\hat{\delta \sigma}^{(2)}=Q_{\mathrm{SM}}^{(2)} \text {. }
$$

In this gauge, Eqs. (196) and (200) reduce, respectively, to

$$
\begin{aligned}
3 H^{2}\left(\hat{A}^{(2)}-2 \hat{A}^{2}\right) & \approx-4 \pi G \hat{\delta \rho}^{(2)}, \\
H \partial_{i}\left(\hat{A}^{(2)}-\frac{3}{2} \hat{A}^{2}\right) & \approx-4 \pi G \hat{\delta}_{i}^{(2)} .
\end{aligned}
$$

Using the first-order constraint equations Eqs. (173) and (177), these can be rewritten as

$$
\hat{A}^{(2)} \approx \frac{1}{3 H^{2}+H^{\prime} / \bar{c}_{s}^{2}}\left[-\frac{\bar{\sigma}^{\prime} \bar{P}_{, X}}{2 \bar{c}_{s}^{2}}\left(Q_{\mathrm{SM}}^{(2) \prime}-\Xi \delta s^{(2)}\right)+\frac{1}{2}\left(\bar{P}_{, \sigma}-\bar{\sigma}^{\prime 2} \bar{P}_{, X \sigma}\right) Q_{\mathrm{SM}}^{(2)}+6\left(\frac{H^{\prime}}{\bar{\sigma}^{\prime}}\right)^{2} Q_{\mathrm{SM}}^{2}-\frac{\hat{\Lambda}_{\rho}}{2}\right]
$$

and

$$
\hat{A}^{(2)} \approx-\frac{H^{\prime}}{H \bar{\sigma}^{\prime}}\left[Q_{\mathrm{SM}}^{(2)}+\frac{\bar{\theta}^{\prime}}{\bar{\sigma}^{\prime}} Q_{\mathrm{SM}} \delta s+\frac{1}{2 \bar{\sigma}^{\prime}}\left(\frac{\bar{\sigma}^{\prime \prime}}{\bar{c}_{s}^{2} \bar{\sigma}^{\prime}}-3 \frac{H^{\prime}}{H}+\frac{\bar{\sigma}^{\prime} \bar{P}_{, X \sigma}}{\bar{P}_{, X}}\right) Q_{\mathrm{SM}}^{2}-\frac{1}{\bar{\sigma}^{\prime}} \partial^{-2} \partial^{i} V_{i}\right] .
$$

This last equation contains a nonlocal term because we have written the momentum constraint as a scalar equation while keeping the second-order vector $V_{i}$ defined in (187). By combining these two relations to get rid of $A^{(2)}$ one obtains the following first integral for $Q_{\mathrm{SM}}^{(2)}$ :

$$
\begin{aligned}
Q_{\mathrm{SM}}^{(2) \prime} & +\left(\frac{H^{\prime}}{H}-\frac{\bar{\sigma}^{\prime \prime}}{\bar{\sigma}^{\prime}}\right) Q_{\mathrm{SM}}^{(2)}-\bar{\Xi} \delta s^{(2)} \\
& \approx\left[3 \bar{\sigma}^{\prime} \bar{P}_{, X} \bar{c}_{s}^{2}-\frac{1}{2 \bar{\sigma}^{\prime}}\left(\frac{\bar{\sigma}^{\prime \prime}}{\bar{c}_{s}^{2} \bar{\sigma}^{\prime}}-3 \frac{H^{\prime}}{H}+\frac{\bar{\sigma}^{\prime} \bar{P}_{, X \sigma}}{\bar{P}_{, X}}\right)\left(3 H \bar{c}_{s}^{2}+\frac{H^{\prime}}{H}\right)\right] Q_{\mathrm{SM}}^{2}-\frac{\bar{c}_{s}^{2} \hat{\Lambda}_{\rho}}{\bar{P}_{, X} \bar{\sigma}^{\prime}} \\
& -\left(3 H \bar{c}_{s}^{2}+\frac{H^{\prime}}{H}\right)\left(\frac{\bar{\theta}^{\prime}}{\bar{\sigma}^{\prime}} Q_{\mathrm{SM}} \delta s-\frac{1}{\bar{\sigma}^{\prime}} \partial^{-2} \partial^{i} V_{i}\right) .
\end{aligned}
$$

This equation is the second-order equivalent of (177). We have put together on the left hand side all the terms depending on purely second-order quantities, and on the right hand side all the terms which are quadratic in first-order quantities. As in the first-order case, the entropy perturbation sources the evolution of the adiabatic perturbation. The nonlocal term containing $V_{i}$ comes from the momentum constraint and is a new feature with respect to the first-order case (or the second-order case for a single scalar field). However, as we have discussed earlier, it becomes quickly negligible on large scales in an expanding universe, in which case the first integral (213) becomes a scalar local equation.

We eventually derive a second-order equation for the entropy mode $\delta s^{(2)}$, which will be second order in time. Since we are restricting ourselves to large scales, we simply expand the spatial components of Eq. (126) up to second order. This gives

$$
\begin{aligned}
& \delta\left(\ddot{s}_{i}\right)^{(2)}+\left(3 H+\frac{\bar{P}_{, X}^{\prime}}{\bar{P}_{, X}}\right) \delta\left(\dot{s}_{i}\right)^{(2)}+\left(\bar{\mu}_{s}^{2}+\frac{\bar{\Xi}^{2}}{\bar{c}_{s}^{2}}\right) \delta s_{i}^{(2)}+\delta\left(\Theta+\frac{\dot{P}_{, X}}{P_{, X}}\right) \partial_{i} \delta s^{\prime} \\
& \quad+\delta\left(\mu_{s}^{2}+\frac{\Xi^{2}}{c_{s}^{2}}\right) \partial_{i} \delta s \approx-\frac{\Xi}{\bar{P} \bar{P}_{, X} \bar{\sigma}^{\prime}} \delta \epsilon_{i}^{(2)},
\end{aligned}
$$

where we have neglected the gradient of the comoving energy density at first order, $\delta \epsilon_{i}^{(1)}$ which according to Eq. (168) is subdominant on large scales.

To proceed, we need the spatial components of the first and second time derivatives of the covectors $s_{a}$. By using Eq. (149) for $s_{i}$ at second order and ignoring the higher-order terms in the gradient expansion, one obtains

$$
\delta\left(\dot{s}_{i}\right)^{(2)} \approx \delta s_{i}^{(2) \prime}-A \partial_{i} \delta s^{\prime}
$$


and, by applying once more (149),

$$
\delta\left(\ddot{s}_{i}\right)^{(2)} \approx \delta s_{i}^{(2) \prime \prime}-A^{\prime} \partial_{i} \delta s^{\prime}-2 A \partial_{i} \delta s^{\prime \prime} .
$$

Recalling that

$$
\delta s_{i}^{(2)}=\partial_{i} \delta s^{(2)}+\frac{\delta \sigma}{\bar{\sigma}^{\prime}} \partial_{i} \delta s^{\prime},
$$

we can then substitute the expressions (189) and (215-216) for the second-order entropy component and its derivatives, and use equation (161) and its time derivative. Defining

$$
M_{I J} \equiv-\frac{\mathcal{D}_{I} \mathcal{D}_{J} P}{P_{, X}}+\dot{\sigma}^{2} R_{I K J L} e_{\sigma}^{K} e_{\sigma}^{L}+\frac{1}{\dot{\sigma}^{2} P_{X}^{2}}\left[\left(2+c_{s}^{2}\right) P_{, I} P_{, J}+c_{s}^{2} \dot{\sigma}^{4} P_{, X I} P_{, X J}-c_{s}^{2} \dot{\sigma}^{2} P_{, X I} P_{J}-c_{s}^{2} \dot{\sigma}^{2} P_{, X J} P_{I}\right]
$$

such that

$$
\mu_{s}^{2}+\frac{\Xi^{2}}{c_{s}^{2}}=e_{s}^{I} e_{s}^{J} M_{I J}
$$

the equation (214) can be written as the spatial gradient of the following scalar equation

$$
\begin{aligned}
& \delta s^{(2)^{\prime \prime}}+\left(3 H+\frac{\bar{P}_{, X}^{\prime}}{\bar{P}_{, X}}\right) \delta s^{(2)^{\prime}}+\left(\bar{\mu}_{s}^{2}+\frac{\bar{\Xi}^{2}}{\bar{c}_{s}^{2}}\right) \delta s^{(2)} \approx-\frac{\bar{\theta}^{\prime}}{\bar{\sigma}^{\prime}} \delta s^{\prime 2}-\left(3 H \frac{\bar{\Xi}}{2 \bar{\sigma}^{\prime}}-\frac{2}{\bar{\sigma}^{\prime}} \bar{e}_{\sigma}^{I} \bar{e}_{s}^{J} \bar{M}_{I J}\right) \delta s \delta s^{\prime} \\
& -\frac{\bar{e}_{s}^{I} \bar{e}_{s}^{J}}{2}\left[\bar{\sigma}^{\prime}\left(\bar{\Xi}-\bar{\theta}^{\prime}\right) \bar{M}_{I J, X}+\bar{e}_{s}^{K} \mathcal{D}_{K} \bar{M}_{I J}-2 \frac{\Xi}{\bar{\sigma}^{\prime}} \bar{M}_{I J}\right] \delta s^{2}-\frac{\bar{\Xi}}{\bar{P}_{, X} \bar{\sigma}^{\prime}} \delta \epsilon^{(2)} .
\end{aligned}
$$

Let us make a few clarifying comments: when calculating the various derivatives of $M_{I J}$ with respect to the fields and to $X, \dot{\sigma}^{2}$ is replaced by $2 X$ and the basis vectors $\bar{e}_{\sigma}^{I}, \bar{e}_{s}^{I}$ are considered as constant (they are not functions of $X$ and the fields); eventually, $\mathcal{D}_{K} \bar{M}_{I J} \equiv \partial_{K} \bar{M}_{I J}-\bar{\Gamma}_{K I}^{L} \bar{M}_{L J}-\bar{\Gamma}_{K J}^{L} \bar{M}_{I L}$.

Note that this equation is closed, in the sense that only the entropy field perturbation appears: even when $\delta \epsilon^{(2)}$ is not negligible, it can be written in terms of $\delta s$ and $\delta s^{\prime}$ by using Eqs. (206) and (187). Thus, on large scales the entropy field evolves independently of the adiabatic components, as in the linear theory.

\subsection{Generalized uniform density and comoving curvature perturbations}

We now derive the large-scale evolution equation for $\zeta^{(2)}$, expanding to second order Eq. (128). In the fluid description, it was shown in $[51,19]$ that on large scales the evolution equation for $\zeta^{(2)}$ can be written as

$$
\zeta^{(2) \prime} \approx-\frac{H}{\bar{\rho}+\bar{P}} \Gamma^{(2)}-\frac{1}{\bar{\rho}+\bar{P}} \Gamma_{1} \zeta_{1}^{\prime},
$$

where $\Gamma^{(2)}$ can be read from the second-order decomposition of the quantity $\Gamma_{a}$ for a fluid, defined in Eq. (40), i.e.,

$$
\delta \Gamma_{i}^{(2)}=\partial_{i} \Gamma^{(2)}+\frac{\delta \rho}{\bar{\rho}^{\prime}} \partial_{i} \Gamma^{(1) \prime} .
$$

In the two-scalar field case considered here we must compare this expansion with the expression for $\Gamma_{a}$ given in the large-scale limit by Eq. (127). Expanding this equation to second order, using Eq. (189), one obtains

$$
\begin{aligned}
\delta \Gamma_{i}^{(2)} & \approx-\delta \epsilon_{i}^{(2)}\left\{\frac{\bar{\sigma}^{\prime}}{\bar{\rho}^{\prime}}\left[\left(1+\bar{c}_{s}^{2}\right) \bar{P}_{, \sigma}-\bar{c}_{s}^{2} \bar{\sigma}^{\prime 2} \bar{P}_{, X \sigma}\right]\right\}+\left(\bar{P}_{, X} \bar{\sigma}^{\prime} \bar{\Xi}\right)\left(\partial_{i} \delta s^{(2)}+\frac{\delta \sigma}{\bar{\sigma}^{\prime}} \partial_{i} \delta s\right) \\
& +\partial_{i} \delta s \delta\left(P_{, X} \dot{\sigma} \Xi\right)
\end{aligned}
$$

where we have used that $\delta \epsilon^{(1)}$ is negligible on large scales. From this, defining $\xi_{I}$ as

$$
\xi_{I} \equiv\left(1+c_{s}^{2}\right) P_{, I}-c_{s}^{2} \dot{\sigma}^{2} P_{, X I}
$$

such that

$$
P_{, X} \dot{\sigma} \Xi=e_{s}^{I} \xi_{I}
$$


one obtains the expression

$$
\Gamma^{(2)} \approx-\bar{e}_{\sigma}^{I} \bar{\xi}_{I}\left(\frac{\bar{\sigma}^{\prime}}{\bar{\rho}^{\prime}} \delta \epsilon^{(2)}+\frac{1}{2 \bar{\sigma}^{\prime}} \delta s \delta s^{\prime}\right)+\bar{e}_{s}^{I} \bar{\xi}_{I} \delta s^{(2)}+\left[\bar{e}_{s}^{I} \bar{e}_{s}^{J} \bar{\xi}_{I ; J}-\bar{\sigma}^{\prime}\left(\bar{\theta}^{\prime}-\bar{\Xi}\right) \bar{e}_{s}^{I} \bar{\xi}_{I, X}\right] \frac{\delta s^{2}}{2} .
$$

From the previous expression it is immediate to rewrite the second-order evolution equation for $\zeta^{(2)}$ on large scales, Eq. (220), as

$$
\zeta^{(2) \prime} \approx-\frac{H}{\bar{P}_{, X} \bar{\sigma}^{\prime 2}}\left[-\bar{e}_{\sigma}^{I} \bar{\xi}_{I}\left(\frac{\bar{\sigma}^{\prime}}{\bar{\rho}^{\prime}} \delta \epsilon^{(2)}+\frac{1}{2 \bar{\sigma}^{\prime}} \delta s \delta s^{\prime}\right)+\bar{e}_{s}^{I} \bar{\xi}_{I} \delta s^{(2)}+\left(\bar{e}_{s}^{I} \bar{e}_{s}^{J} \bar{\xi}_{I ; J}-\bar{\sigma}^{\prime}\left(\bar{\theta}^{\prime}-\bar{\Xi}\right) \bar{e}_{s}^{I} \bar{\xi}_{I, X}-2 \bar{P}_{, X} \bar{\Xi}^{2}\right) \frac{\delta s^{2}}{2}\right] .
$$

The previous expression shows that the second-order curvature perturbation is, as expected, sourced only by entropy modes on large scales. On the other hand, it depends in a much richer way on $\xi_{I}$ and on the derivatives of $P$, that is on the form of the kinetic terms, than its linear counterpart in eq. (181). Moreover, $\xi_{I}$ reduces to $-2 V_{, I}$ for a standard Lagrangian, with no dependence on the kinetic term $X$. The appearence of $\bar{\xi}_{, I X}$ on the right hand side of (226), acting as a source for the large-scale evolution of the curvature perturbation, is thus solely due to the non-standard nature of the Lagrangian we are considering. It is important to emphasize that the nonlinear formalism we adopted, as well as the fact that we considered a very general Lagrangian, have allowed us to straightforwardly obtain a relatively elegant and compact expression. The same would be difficult to get working directly in a coordinate based approach, or considering a specific Lagrangian.

It is also useful to express our results in terms of $\mathcal{R}_{a}$. The spatial components of $\mathcal{R}_{a}$ can be decomposed as

$$
\mathcal{R}_{i}^{(2)}=\partial_{i} \mathcal{R}^{(2)}+\frac{\delta \sigma}{\bar{\sigma}^{\prime}} \partial_{i} \mathcal{R}^{(1) \prime}-\frac{H}{\bar{\sigma}^{\prime 2}} V_{i},
$$

with

$$
\mathcal{R}^{(2)} \equiv-\delta \alpha^{(2)}+\frac{H}{\bar{\sigma}^{\prime}} \delta \sigma^{(2)}+\frac{\delta \sigma}{\bar{\sigma}^{\prime}}\left[-\mathcal{R}^{(1) \prime}+\frac{1}{2}\left(\frac{H}{\bar{\sigma}^{\prime}}\right)^{\prime} \delta \sigma+\bar{\theta}^{\prime} \frac{H}{\bar{\sigma}^{\prime}} \delta s\right] .
$$

The last term in Eq. (227) comes from the fact that, like $\epsilon_{a}$ and in contrast to $\zeta_{a}, \mathcal{R}_{a}$ is defined in terms of the spatial momentum which cannot be expressed in general as a pure gradient. When this term can be neglected, and only then, $\mathcal{R}^{(2)}$ coincides with the second-order comoving curvature perturbation defined in [52, 53].

It is easy to derive a first-order (in time) evolution equation for $\mathcal{R}^{(2)}$ by noting that $\zeta^{(2)}$ and $\mathcal{R}^{(2)}$ are related on large scales. Indeed, expanding Eq. (138) to second order using (184) and (227), and neglecting terms proportional to $\delta \epsilon^{(1)}$, one gets

$$
\zeta^{(2)}+\mathcal{R}^{(2)} \approx \frac{1}{3 \bar{P}_{, X} \bar{\sigma}^{\prime 2}} \delta \epsilon^{(2)} .
$$

When $\delta \epsilon^{(2)}$ is negligible on large scales, like in an expanding universe where we can neglect $V_{i}, \zeta^{(2)}$ and $\mathcal{R}^{(2)}$ coincide on large scales as in the single-field case [53]. However, this is not true in general in the multi-field case if $V_{i}$ cannot be neglected.

From this relation and the evolution equation of $\zeta^{(2)}$, Eq. (226), one can find a large-scale evolution equation for $\mathcal{R}^{(2)}$,

$\mathcal{R}^{(2) \prime} \approx \frac{H}{\bar{P}_{, X} \bar{\sigma}^{\prime 2}}\left[-\frac{\bar{e}_{\sigma}^{I} \bar{\xi}_{I}}{2 \bar{\sigma}^{\prime}} \delta s \delta s^{\prime}+\bar{e}_{s}^{I} \bar{\xi}_{I} \delta s^{(2)}+\left(\bar{e}_{s}^{I} \bar{e}_{s}^{J} \bar{\xi}_{I ; J}-\bar{\sigma}^{\prime}\left(\bar{\theta}^{\prime}-\bar{\Xi}\right) \bar{e}_{s}^{I} \bar{\xi}_{I, X}-2 \bar{P}_{, X} \bar{\Xi}^{2}\right) \frac{\delta s^{2}}{2}+\left(\bar{c}_{s}^{2}+\frac{H^{\prime}}{3 H^{2}}\right) \delta \epsilon^{(2)}\right]$.

The second-order uniform adiabatic field perturbation $\mathcal{R}^{(2)}$ can be related on large scales to $Q_{\mathrm{SM}}^{(2)}$, by combining Eqs. (194) and (228). One obtains

$$
\mathcal{R}^{(2)} \approx \frac{H}{\bar{\sigma}^{\prime}}\left[Q_{\mathrm{SM}}^{(2)}-\frac{1}{\bar{\sigma}^{\prime}}\left(Q_{\mathrm{SM}}^{\prime}-\bar{\theta}^{\prime} \delta s\right) Q_{\mathrm{SM}}-\frac{1}{2 H}\left(\frac{H}{\bar{\sigma}^{\prime}}\right)^{\prime} Q_{\mathrm{SM}}^{2}\right],
$$

which can be used, together with the linear first integral (177), to show that Eq. (230) is equivalent to the first integral (213). 


\section{Conclusions}

In this work, we adopted a covariant formalism to derive exact evolution equations for nonlinear perturbations, in a universe dominated by two scalar fields. These scalar fields are characterized by non-canonical kinetic terms and an arbitrary field space metric, a situation typically encountered in inflationary models inspired by string theory. Our exact equations can be expressed in a relatively compact way, and in physically interesting limits they closely resemble their linear counterpart. They acquire a quite simple and elegant form, due to the fact that we considered a very general Lagrangian to perform our calculations.

Extending the methods of [21] to a general field space metric, we have decomposed our non-linear scalar perturbations into adiabatic and entropy modes, corresponding to a generalization of analogous definitions adopted in the linear theory. Then, we derived the corresponding evolution equations, that acquire several new contributions associated with the non-canonical kinetic terms for the scalar fields. We also obtained a nonlinear generalization of the curvature perturbation on uniform energy density hypersurfaces, showing that on large scales it is sourced only by the nonlinear entropy perturbation.

We have used these nonlinear equations as a starting point to show how to extend and generalize some results previously obtained in the literature, in a relatively straightforward way. In particular, we went beyond previous works by computing explicitly the evolution of the second-order adiabatic and entropy components on large scales, in the case in which the scalar fields have non-canonical kinetic terms. The second-order adiabatic component is, in this limit, governed by a local first-order (in time) evolution equation, sourced by terms depending on the second-order entropy perturbation as well as, quadratically, on the first-order entropy perturbation. Both first and second order entropy perturbations satisfy a second order (in time) evolution equation and the full system of equations, valid on large scales, is thus closed. The final system of equations enables one to clearly identify new effects due to the non-canonical structure of the scalar fields Lagrangian. A representative example is equation (226), that governs the second-order evolution equation for the curvature perturbation on large scales. This quantity is sourced by combinations of first and second-order entropy perturbations, with coefficients depending on the form of the kinetic terms. As discussed in Sec. 7.2, in some situations, cancellations can occur, reducing the size of some coefficients with respect to the others. In these cases, the curvature pertubation would be sensitive only to some type of terms, with important consequences for the evolution of second-order perturbations and thus for non-Gaussianities. All the results derived on large scales in Sec. 7 are applicable to the interesting scenario of multifield DBI inflation of the type studied in $[12,13]$.

In conclusions, we have shown that our results, and more in general the covariant approach to the dynamics of fluctuations, can be important to analyze the evolution of perturbations in multi-field models of inflation inspired by high-energy physics or string theory. This interesting subject will offer, in the near future, unique opportunities to allow comparisons between predictions of high-energy physics motivated inflationary models, and observations of the cosmic microwave background radiation.

\section{Acknowledgments}

We thank David Langlois, Danièle A. Steer and Filippo Vernizzi for very useful discussions and for their careful reading of the draft. We also would like to thank Jean-Luc Lehners for pointing out a typo in Eq. (195). Most of this work has been done while G. T. was partially supported by MEC and FEDER under grant FPA2006-05485, by CAM under grant HEPHACOS P-ESP-00346, and by the UniverseNet network (MRTN-CT-2006-035863). He thanks the kind hospitality of APC, Paris, where this work was initiated.

\section{A Useful identities in a two-field system}

\section{A.1 Covariant identities}

$$
\begin{aligned}
D_{a} \dot{\sigma} & =\dot{\sigma}_{a}+\ddot{\sigma} u_{a}-\dot{\theta} s_{a}=\dot{\sigma}_{a}^{\perp}-\dot{\theta} s_{a}, \\
D_{a} X & =\dot{\sigma} D_{a} \dot{\sigma}-\frac{1}{2} D_{a} \Pi, \\
D_{a} P_{, X} & =P_{, X X} D_{a} X+P_{, X \sigma} \sigma_{a}^{\perp}+P_{, X s} s_{a},
\end{aligned}
$$




$$
\begin{aligned}
\dot{P}_{, X} & =P_{, X X}\left[\dot{\sigma} \ddot{\sigma}-\frac{1}{2} \dot{\Pi}\right]+P_{, X \sigma} \dot{\sigma} \\
D_{a} P_{, X X} & =P_{, X X X} D_{a} X+P_{, X X \sigma} \sigma_{a}^{\perp}+P_{, X X s} s_{a} \\
D_{a} P_{, X \sigma} & =P_{, X X \sigma} D_{a} X+P_{, X \sigma \sigma} \sigma_{a}^{\perp}+P_{, X \sigma s} s_{a} \\
D_{a} \dot{P}_{, X} & =\left(D_{a} P_{, X X}\right)\left[\dot{\sigma} \ddot{\sigma}-\frac{1}{2} \dot{\Pi}\right]+D_{a}\left(P_{, X \sigma} \dot{\sigma}\right) \\
& +P_{, X X}\left[\left(D_{a} \dot{\sigma}\right) \ddot{\sigma}-\frac{1}{2} D_{a} \dot{\Pi}\right]+P_{, X X} \dot{\sigma}\left[\ddot{\sigma}_{a}^{\perp}-\ddot{\theta} s_{a}-\dot{\theta} \dot{s}_{a}\right]
\end{aligned}
$$

\section{A.2 Background identities}

$$
\begin{gathered}
H^{\prime}=-4 \pi G \bar{P}_{, X} \bar{\sigma}^{\prime 2} \\
\bar{P}_{, \sigma}^{\prime}=\bar{\sigma}^{\prime}\left(\bar{P}_{, \sigma \sigma}+\bar{\sigma}^{\prime \prime} \bar{P}_{, X \sigma}\right)+\bar{\theta}^{\prime} \bar{P}_{, s}, \\
\bar{P}_{, s}^{\prime}=\bar{\sigma}^{\prime}\left(\bar{P}_{, \sigma s}+\bar{\sigma}^{\prime \prime} \bar{P}_{, X s}\right)-\bar{\theta}^{\prime} \bar{P}_{, \sigma}, \\
\bar{P}_{, \sigma s}^{\prime}=\bar{\sigma}^{\prime}\left(\bar{P}_{, \sigma \sigma s}+\bar{\sigma}^{\prime \prime} \bar{P}_{, X \sigma s}\right)+\bar{\theta}^{\prime}\left(\bar{P}_{, s s}-\bar{P}_{, \sigma \sigma}\right), \\
\bar{P}_{, s s}^{\prime}=\bar{\sigma}^{\prime}\left(\bar{P}_{, \sigma s s}+\bar{\sigma}^{\prime \prime} \bar{P}_{, X s s}\right)-2 \bar{\theta}^{\prime} \bar{P}_{, \sigma s}, \\
\bar{P}_{, X}^{\prime}=\bar{\sigma}^{\prime}\left(\bar{P}_{, X \sigma}+\bar{\sigma}^{\prime \prime} \bar{P}_{, X X}\right) \\
\bar{P}_{, X X}^{\prime}=\bar{\sigma}^{\prime}\left(\bar{P}_{, X X \sigma}+\bar{\sigma}^{\prime \prime} \bar{P}_{, X X X}\right) \\
\bar{P}_{, X \sigma}^{\prime}=\bar{\sigma}^{\prime}\left(\bar{P}_{X \sigma \sigma}+\bar{\sigma}^{\prime \prime} \bar{P}_{X X \sigma}\right)+\bar{\theta}^{\prime} \bar{P}_{, X s}, \\
\bar{P}_{, X s}^{\prime}=\bar{\sigma}^{\prime}\left(\bar{P}_{, X \sigma s}+\bar{\sigma}^{\prime \prime} \bar{P}_{, X X s}\right)-\bar{\theta}^{\prime} \bar{P}_{, X \sigma},
\end{gathered}
$$

\section{A.3 First-order identities}

$$
\begin{aligned}
\delta e_{\sigma}^{I} & =\frac{1}{\bar{\sigma}^{\prime}}\left(\delta s^{\prime}+\bar{\theta}^{\prime} \delta \sigma\right) \bar{e}_{s}^{I}-\bar{\Gamma}_{J K}^{I} \bar{e}_{\sigma}^{J}\left(\bar{e}_{\sigma}^{K} \delta \sigma+\bar{e}_{s}^{K} \delta s\right), \\
\delta e_{s}^{I} & =-\frac{1}{\bar{\sigma}^{\prime}}\left(\delta s^{\prime}+\bar{\theta}^{\prime} \delta \sigma\right) \bar{e}_{\sigma}^{I}-\bar{\Gamma}_{J K}^{I} \bar{e}_{s}^{J}\left(\bar{e}_{\sigma}^{K} \delta \sigma+\bar{e}_{s}^{K} \delta s\right), \\
\delta(\dot{\sigma}) & =\delta \sigma^{\prime}-\bar{\theta}^{\prime} \delta s-\bar{\sigma}^{\prime} A \\
\delta P_{, s} & =\bar{P}_{, s \sigma} \delta \sigma+\bar{P}_{, s s} \delta s+\bar{P}_{, X s}\left(\bar{\sigma}^{\prime} \delta(\dot{\sigma})-\frac{1}{2} \delta \Pi\right)-\frac{\bar{P}_{, \sigma}}{\bar{\sigma}^{\prime}}\left(\delta s^{\prime}+\bar{\theta}^{\prime} \delta \sigma\right), \\
\delta P_{, X s} & =\bar{P}_{, X s \sigma} \delta \sigma+\bar{P}_{, X s s} \delta s+\bar{P}_{, X X s}\left(\bar{\sigma}^{\prime} \delta(\dot{\sigma})-\frac{1}{2} \delta \Pi\right)-\frac{\bar{P}_{, X \sigma}}{\bar{\sigma}^{\prime}}\left(\delta s^{\prime}+\bar{\theta}^{\prime} \delta \sigma\right), \\
\delta P_{s s} & =\bar{P}_{, s s \sigma} \delta \sigma+\bar{P}_{, s s s} \delta s+\bar{P}_{, X s s}\left(\bar{\sigma}^{\prime} \delta(\dot{\sigma})-\frac{1}{2} \delta \Pi\right)-2 \frac{\bar{P}_{, s \sigma}}{\bar{\sigma}^{\prime}}\left(\delta s^{\prime}+\bar{\theta}^{\prime} \delta \sigma\right), \\
\delta P_{, X} & =\bar{P}_{, X \sigma} \delta \sigma+\bar{P}_{, X s} \delta s+\bar{P}_{, X X}\left(\bar{\sigma}^{\prime} \delta(\dot{\sigma})-\frac{1}{2} \delta \Pi\right), \\
\delta \dot{P}_{, X} & =\left(\delta P_{, X}\right)^{\prime}-A \bar{P}_{, X} .
\end{aligned}
$$




\section{References}

[1] L. McAllister and E. Silverstein, Gen. Rel. Grav. 40 (2008) 565 [arXiv:0710.2951 [hep-th]]; C. P. Burgess, PoS P2GC, 008 (2006) [Class. Quant. Grav. 24, S795 (2007)] [arXiv:0708.2865 [hep-th]].

[2] E. Silverstein and D. Tong, Phys. Rev. D 70 (2004) 103505 [arXiv:hep-th/0310221].

[3] M. Alishahiha, E. Silverstein and D. Tong, Phys. Rev. D 70 (2004) 123505 [arXiv:hep-th/0404084].

[4] X. Chen, Phys. Rev. D 71, 063506 (2005) [arXiv:hep-th/0408084].

[5] X. Chen, JHEP 0508, 045 (2005) [arXiv:hep-th/0501184].

[6] C. Armendariz-Picon, T. Damour and V. F. Mukhanov, Phys. Lett. B 458 (1999) 209 [arXiv:hepth/9904075]; J. Garriga and V. F. Mukhanov, Phys. Lett. B 458 (1999) 219 [arXiv:hep-th/9904176].

[7] X. Chen, M. x. Huang, S. Kachru and G. Shiu, JCAP 0701 (2007) 002 [arXiv:hep-th/0605045].

[8] D. Easson, R. Gregory, G. Tasinato and I. Zavala, JHEP 0704 (2007) 026 [arXiv:hep-th/0701252].

[9] D. A. Easson, R. Gregory, D. F. Mota, G. Tasinato and I. Zavala, JCAP 0802 (2008) 010 [arXiv:0709.2666 [hep-th]].

[10] M. x. Huang, G. Shiu and B. Underwood, Phys. Rev. D 77, 023511 (2008) [arXiv:0709.3299 [hep-th]].

[11] D. Langlois and S. Renaux-Petel, JCAP 0804 (2008) 017 [arXiv:0801.1085 [hep-th]].

[12] D. Langlois, S. Renaux-Petel, D. A. Steer and T. Tanaka, Phys. Rev. Lett. 101 (2008) 061301 [arXiv:0804.3139 [hep-th]].

[13] D. Langlois, S. Renaux-Petel, D. A. Steer and T. Tanaka, Phys. Rev. D 78, 063523 (2008) arXiv:0806.0336 [hep-th].

[14] X. Gao, JCAP 0806 (2008) 029 [arXiv:0804.1055 [astro-ph]].

[15] F. Arroja, S. Mizuno and K. Koyama, JCAP 0808 (2008) 015 [arXiv:0806.0619 [astro-ph]].

[16] Y. F. Cai and W. Xue, arXiv:0809.4134 [hep-th].

[17] D. Langlois, Phys. Rev. D 59, 123512 (1999) [arXiv:astro-ph/9906080].

[18] D. Langlois and F. Vernizzi, Phys. Rev. Lett. 95, 091303 (2005) [arXiv:astro-ph/0503416].

[19] D. Langlois and F. Vernizzi, Phys. Rev. D 72, 103501 (2005) [arXiv:astro-ph/0509078].

[20] D. Langlois and F. Vernizzi, JCAP 0602, 014 (2006) [arXiv:astro-ph/0601271].

[21] D. Langlois and F. Vernizzi, JCAP 0702 (2007) 017 [arXiv:astro-ph/0610064].

[22] G. F. R. Ellis and M. Bruni, Phys. Rev. D 40, 1804 (1989).

[23] S. W. Hawking, Astrophys. J. 145, 544 (1966).

[24] M. Bruni, G. F. R. Ellis and P. K. S. Dunsby, Class. Quant. Grav. 9, 921 (1992).

[25] C. Gordon, D. Wands, B. A. Bassett and R. Maartens, Phys. Rev. D 63 (2001) 023506 [arXiv:astro$\mathrm{ph} / 0009131]$.

[26] D. Wands, Lect. Notes Phys. 738 (2008) 275 [arXiv:astro-ph/0702187].

[27] R. M. Wald, "General Relativity,", Chicago University Press, Usa (1984).

[28] D. H. Lyth, K. A. Malik and M. Sasaki, JCAP 0505 (2005) 004 [arXiv:astro-ph/0411220]. 
[29] S. Groot Nibbelink and B. J. W. van Tent, arXiv:hep-ph/0011325; S. Groot Nibbelink and B. J. W. van Tent, Class. Quant. Grav. 19, 613 (2002) [arXiv:hep-ph/0107272].

[30] F. Di Marco, F. Finelli and R. Brandenberger, Phys. Rev. D 67 (2003) 063512 [arXiv:astro-ph/0211276].

[31] G. I. Rigopoulos, E. P. S. Shellard and B. W. van Tent, Phys. Rev. D 73, 083521 (2006) [arXiv:astro$\mathrm{ph} / 0504508]$.

[32] D. S. Salopek and J. R. Bond, Phys. Rev. D 42, 3936 (1990).

[33] G. L. Comer, N. Deruelle, D. Langlois and J. Parry, Phys. Rev. D 49 (1994) 2759.

[34] N. Deruelle and D. Langlois, Phys. Rev. D 52, 2007 (1995) [arXiv:gr-qc/9411040].

[35] Y. i. Takamizu and S. Mukohyama, arXiv:0810.0746 [gr-qc].

[36] M. Sasaki and E. D. Stewart, Prog. Theor. Phys. 95, 71 (1996) [arXiv:astro-ph/9507001].

[37] A. A. Starobinsky, JETP Lett. 42, 152 (1985) [Pisma Zh. Eksp. Teor. Fiz. 42, 124 (1985)].

[38] M. Sasaki, Prog. Theor. Phys. 76, 1036 (1986); V. F. Mukhanov, Zh. Éksp. Teor. Fiz. 94, 1 (1988) [Sov. Phys. JETP 68, 1297 (1988)].

[39] J. M. Bardeen, Phys. Rev. D 22, 1882 (1980).

[40] J. M. Bardeen, P. J. Steinhardt and M. S. Turner, Phys. Rev. D 28, 679 (1983).

[41] K. A. Malik and D. Wands, arXiv:0809.4944 [astro-ph].

[42] K. A. Malik, JCAP 0511, 005 (2005) [arXiv:astro-ph/0506532].

[43] K. Enqvist and A. Vaihkonen, JCAP 0409, 006 (2004) [arXiv:hep-ph/0405103].

[44] A. Jokinen and A. Mazumdar, JCAP 0604, 003 (2006) [arXiv:astro-ph/0512368].

[45] N. Barnaby and J. M. Cline, Phys. Rev. D 73, 106012 (2006) [arXiv:astro-ph/0601481].

[46] D. H. Lyth and Y. Rodriguez, Phys. Rev. D 71, 123508 (2005) [arXiv:astro-ph/0502578]; ibid., Phys. Rev. Lett. 95, 121302 (2005) [arXiv:astro-ph/0504045].

[47] F. Vernizzi and D. Wands, JCAP 0605, 019 (2006) [arXiv:astro-ph/0603799].

[48] D. Langlois, F. Vernizzi and D. Wands, arXiv:0809.4646 [astro-ph].

[49] M. Kawasaki, K. Nakayama, T. Sekiguchi, T. Suyama and F. Takahashi, arXiv:0808.0009 [astro-ph].

[50] M. Kawasaki, K. Nakayama, T. Sekiguchi, T. Suyama and F. Takahashi, arXiv:0810.0208 [astro-ph].

[51] K. A. Malik and D. Wands, Class. Quant. Grav. 21, L65 (2004) [arXiv:astro-ph/0307055].

[52] J. M. Maldacena, JHEP 0305, 013 (2003) [arXiv:astro-ph/0210603].

[53] F. Vernizzi, Phys. Rev. D 71, 061301 (2005) [arXiv:astro-ph/0411463]. 